Draft VERSiOn MARCH 28, 2014

Preprint typeset using $\mathrm{AT}_{\mathrm{E} X} \mathrm{X}$ style emulateapj v. 12/16/11

\title{
THE MCGILL MAGNETAR CATALOG
}

\author{
S. A. Olausen \& V. M. Kaspi \\ Department of Physics, Rutherford Physics Building, McGill University, 3600 University Street, Montreal, Quebec, H3A 2T8, Canada \\ Draft version March 28, 2014
}

\begin{abstract}
We present a catalog of the 26 currently known magnetars and magnetar candidates. We tabulate astrometric and timing data for all catalog sources, as well as their observed radiative properties, particularly the spectral parameters of the quiescent X-ray emission. We show histograms of the spatial and timing properties of the magnetars, comparing them with the known pulsar population, and we investigate and plot possible correlations between their timing, X-ray, and multiwavelength properties. We find the scale height of magnetars to be in the range 20-31 pc, assuming they are exponentially distributed. This range is smaller than that measured for OB stars, providing evidence that magnetars are born from the most massive O stars. From the same fits, we find that the Sun lies $\sim 13-22$ pc above the Galactic plane, consistent with previous measurements. We confirm previously identified correlations between quiescent X-ray luminosity $L_{\mathrm{X}}$ and magnetic field $B$, as well as X-ray spectral power-law index $\Gamma$ and $B$, and show evidence for an excluded region in a plot of $L_{\mathrm{X}} \mathrm{vs} . \Gamma$. We also present an updated $k T$ versus characteristic age plot, showing magnetars and high- $B$ radio pulsars are hotter than lower- $B$ neutron stars of similar age. Finally, we observe a striking difference between magnetars detected in the the hard X-ray and radio bands; there is a clear correlation between the hard and soft X-ray flux, whereas the radio-detected magnetars all have low soft X-ray flux suggesting, if anything, that the two bands are anti-correlated.
\end{abstract}

An online version of the catalog is located at http://www.physics.mcgill.ca/ pulsar/magnetar/main.html. Keywords: catalogs — pulsars: general — stars: magnetars — stars: neutron

\section{INTRODUCTION}

The class of neutron stars today identified as "magnetars" was first noted in 1979 with the detection of repeated bursts by space-based hard Xray/soft gamma-ray instruments (Mazets et al. 1979b,a; Mazets \& Golenetskii 1981). Though originally thought to have the same origin as the classical gamma-ray bursts (GRBs), repeated bursts, including one enormous flare on 1979 March 5, from the direction of the star-forming Dorado region in the Large Magellanic Cloud (Mazets et al. 1979b) as well as from what today is known to be magnetar SGR 1900+14 (Mazets et al. 1979a; Mazets \& Golenetskii 1981), provided an important distinction and hint of a new class of Galactic sources. The repeated bursts had somewhat softer spectra than those of most GRBs, hence the sources' designation as 'Soft Gamma Repeaters' (SGRs). The 8-s pulsations seen in the declining flux tail following the large flare were strongly suggestive of a neutron-star origin for SGRs. That these two sources truly represented a distinct class of gamma-ray bursters was more fully recognized in 1983 when a third Galactic source, SGR 1806-20, underwent a major burst episode (Laros et al. 1987). Both Galactic sources were noted to be very close to the Galactic Plane, suggesting youth, a conclusion supported by the coincidence of the LMC source with the supernova remnant N49 (Cline et al.|1982).

Meanwhile, Fahlman \& Gregory (1981) reported an unusual 7-s X-ray pulsar, 1E $2259+586$, in the Galactic supernova remnant CTB 109. Originally thought to be a low-mass X-ray binary albeit without any obvi-

Electronic address: scott.olausen@mail.mcgill.ca

Electronic address: vkaspi@physics.mcgill.ca ous companion, the source was soon recognized as being similar to a handful of other 'anomalous' sources (including 4U 0142+61 and 1E 1048.1-5937) (see Hellier 1994; Duncan \& Thompson 1996; van Paradijs et al. 1995; (Mereghetti \& Stella 1995), distinguished by their bright X-ray pulsations at few-second periods, X-ray luminosities far greater than could be explained via rotation power, but no apparent companions from which to accrete. These distinctions led to the sources being termed 'Anomalous X-ray Pulsars' (AXPs) and this descriptor has stuck.

Duncan \& Thompson (1992) proposed that very strongly magnetized neutron stars could be the origin of SGR emission, thereby coining the term 'magnetar.' Thompson \& Duncan (1995) demonstrated that many SGR phenomena are readily explained by a model in which spontaneous magnetic field decay serves as an energy source for both the bursts and any persistent emission. They cited not only energetics arguments but also the need for a high $B$ field to spin down a young neutron star from tens to hundreds of ms (thought to be the typical birth spin period range) to several seconds, within a supernova remnant lifetime. Thompson \& Duncan (1996) further argued that AXPs are also magnetars, with their X-ray luminosities powered by magnetic field decay. The subsequent direct detection of spin-down in an SGR at a rate consistent with the model prediction (Kouveliotou et al. 1998) was a powerful confirmation of the magnetar picture. The detection of SGR-like bursts from two AXPs (Gavriil et al. 2002; Kaspi et al. 2003) unified AXPs and SGRs observationally, as predicted by Thompson \& Duncan (1996). Since then, the distinction between AXPs and SGRs has been further blurred, with practically all sources 
having shown characteristics of both: bursting has now been shown to be a generic behavior of so-called AXPs (e.g. Gavriil et al. 2004; Woods et al. 2005; Kaneko et al. 2010; Scholz \& Kaspi 2011) and AXP-like behavior (namely, absence of bursts for long periods) has been seen in objects previously deemed SGRs, including the original LMC SGR (Kaplan et al. 2001). It is clear that there exists a continuous spectrum of behavior, ranging from anomalously high quiescent X-ray luminosity to occasional bursting and major flaring, in the single class of objects we now call magnetars. This is the conclusion we adopt in this paper. Several authors have written important review papers on magnetars, their observational properties, and outstanding questions in the field; (see Woods \& Thompson 2006; Mereghetti 2008; Kaspi 2010; Rea \& Esposito 2011; Mereghetti 2013). We note that some alternative models for AXPs and SGRs have been proposed, including a fall-back disk model that has the sources accreting from surrounding debris (e.g. Ertan et al. 2007, 2009), a massive white dwarf model (e.g. Malheiro et al. 2012), and also a quark nova model (Ouyed et al. 2007a,b). Although these models are interesting and have their merits, the current evidence to support these pictures for the overall magnetar population is weak; however, they may be relevant in describing certain outlier objects. We consider them no further here but refer the interested reader to the above references.

With the number of identified magnetars and magnetar candidates having grown to over two dozen today, the time is ripe for a systematic compilation of these objects, in the form of the first magnetar catalog, presented here. Specifically we have collected and compiled a wide variety of information on the 21 confirmed and 5 unconfirmed magnetars, including their spatial, spin, and radiative properties across the EM spectrum. Our hope is that this catalog serves as a useful resource to the magnetar-interested community, and ultimately helps to identify and highlight important population properties that could help answer some of the outstanding questions in magnetar physics. Accompanying this paper is a fully referenced and linked online version ${ }^{1}$ which is regularly maintained. We note that Manchester et al. (2005) include magnetars in their online and published radio pulsar catalog 2 , however the information compiled there is basic and restricted for the most part to spatial spin and radio properties.

In $\S 2$ we present the catalog in the form of seven data tables separated by topic. In $\S 3$ we provide analysis and discussion of the magnetar population based on our catalogued data. Finally, concluding remarks are given in $\S 4$.

\section{DATA TABLES}

\subsection{Table 1: Positions and Proper Motions}

In Table 1 we list the astrometric parameters of the catalogued magnetars. These include the right ascension and declination (J2000.0 epoch), the Galactic longitude $l$ and latitude $b$, and the proper motion $\mu$ in RA and Dec. Measurements of distances to the magnetars are listed in Table 7.

\footnotetext{
1 http://www .physics.mcgill.ca/ pulsar/magnetar/main.html

2 http://www.atnf.csiro.au/research/pulsar/psrcat/
}

The positions listed in this Table are generally those from the literature with the smallest reported uncertainties. The uncertainties are unchanged from the original papers and typically, but not necessarily, represent $90 \%$ confidence intervals. In most cases the listed position is from a Chandra observation of the persistent X-ray source, or Swift/X-ray Telescope (XRT) in the case of Swift J1822.3-1606. The exceptions are 4U 0142+61 and SGR 1806-20, where the position is of an optical counterpart, and 1E 1547.0-5408, SGR J1745-2900, XTE J1810-197, and SGR 1900+14, whose listed positions are of radio counterparts. Finally, the five candidate magnetars have no confirmed counterparts at any wavelength, so we list either the best position of the observed bursts or, in the case of AX J1818.8-1559 and AX J1845.0-0258, the Chandra position of the unconfirmed persistent X-ray counterpart.

Unlike positions, all of the tabulated proper motion measurements or upper limits were found in the radio (1E 1547.0-5408 and XTE J1810-197) and optical (4U 0142+61, SGR 1806-20, 1E 1841-045, SGR 1900+14, and 1E 2259+586) bands (but see Kaplan et al. 2009b for proper motion upper limits found in X-ray with Chan$d r a)$. The optical measurements are all corrected for Galactic rotation, whereas the radio ones are not. We also caution that the proper motion measurement of SGR $1900+14$ is of its unconfirmed optical counterpart (see Table 4).

\subsection{Table 2: Timing Properties}

Table 2 contains timing parameters for all catalogued magnetars for which they are available. Specifically, we tabulate the period $P$ and the epoch at which it was measured, the period derivative $\dot{P}$ and the range over which it was measured, the method of measuring $\dot{P}$ (see below), and three physical properties inferred from $P$ and $\dot{P}$, namely, the surface dipolar magnetic field strength $B$, defined as $B=3.2 \times 10^{19}(P \dot{P})^{1 / 2} G$; the spindown luminosity $\dot{E}$, defined as $\dot{E}=4 \pi^{2} I \dot{P} / P^{3}$, where the moment of inertia $I$ is assumed to be $10^{45} \mathrm{~g} \mathrm{~cm}^{2}$; and the characteristic age $\tau_{c}$, defined as $\tau_{c}=P / 2 \dot{P}$. Note that the expression for $B$ assumes simple vacuum dipole radiation and ignores the potentially important torques due to magnetospheric variability and the internal superfluid, both of which have been proposed to be relevant to magnetars (Kaspi et al. 2003; Dib et al. 2009; Archibald et al. 2013; Thompson et al. 2002; Beloborodov 2009).

The values of $\dot{P}$ were found using one of two methods. In the first case (denoted in Table 2 by A) $\dot{P}$ is a long-term average, calculated by fitting a slope to two or more individual measurements of the period. This was done for sources with only sparse timing data or, in the cases of $1 \mathrm{E} 1048.1-5937,1 \mathrm{E} 1547.0-5408$, and SGR 1806-20, for sources with large variations in $\dot{P}$. In the second case (E, ED), $\dot{P}$ was taken from a phase-coherent timing ephemeris that spans the specified range. If the ephemeris has higher-order derivatives (denoted by ED), then the listed value of $\dot{P}$ is only accurate at the period epoch; otherwise $\dot{P}$ is valid over the entire range. For sources where multiple phase-coherent timing solu- 
tions were found in the literature, we generally chose the solution from the most recent refereed publication that covered the most recent glitch-free interval of time, preferring solutions that covered at least several months. If a publication presented multiple timing solutions covering the same interval, we selected the solution that was preferred by the authors. In all cases, see the references provided for details.

\subsection{Table 3: Quiescent Soft X-ray Properties}

This Table contains the soft X-ray properties of catalog magnetars in quiescence. To facilitate cross-source comparisons, we generally report only the phenomenological parameters of an absorbed blackbody plus powerlaw model, although in several cases only one of these two components is required. The columns provided are the neutral hydrogen column density $N_{\mathrm{H}}$, spectral photon index $\Gamma$, blackbody temperature $k T$, a second blackbody temperature $k T_{2}$ (only used for CXOU J010043.1-721134, for which a blackbody plus power law was a poor fit to the data), and the absorbed and unabsorbed fluxes as well as the energy range over which they were derived. We also include a column for the 2 $10 \mathrm{keV}$ unabsorbed flux, which was estimated with the WebPIMMS tool ${ }^{3}$ in cases where the reference gave only absorbed flux or flux in a different energy range. X-ray luminosities are reported in Table 7.

The tabulated parameters generally differ in the various papers in the literature for any given source, so the following explains our procedure in selecting which properties to catalog. We selected parameters from publications in which the reported source flux was historically lowest, in order to ensure as much as possible that the source was truly in quiescence. In cases where there were multiple publications with equivalently low flux, we report those model parameters that had the smallest uncertainties unless more recent observations appeared more reliable, e.g. were able to better disentangle potentially contaminating supernova remnant emission. For a majority of the sources this resulted in the use of spectral parameters obtained from $X M M-N e w t o n$ data, although in several cases the results are taken from Chandra (1E 1048.1-5937, Swift J1834.9-0846, AX J1845.0-0258, and SGRs 0526-66, 1627-41, and J1745-2900) or archival ROSAT (SGR 0501+4516, XTE J1810-197, and Swift J1822.3-1606) data instead. The only other exception is SGR 1806-20 for which we use a model fit derived from simultaneous Suzaku and XMM observations. We caution that in general the stated uncertainties, statistical in nature, may be smaller than the systematic uncertainties due to calibration and cross-calibration issues; for this reason reported parameters may not be optimal when considering data from a different telescope even in the absence of source variability.

There are a few caveats we must make with regards to the flux values listed in Table 3. First, although we do list the lowest reported flux for PSR J1622-4950, it is not clear whether the source had reached quiescence during that observation or whether it was still fading. Hence, the value we report may be an over-estimation of its true quiescent flux. Also, note the upper limit

\footnotetext{
3 http://heasarc.gsfc.nasa.gov/Tools/w3pimms.html
}

for the $2-10 \mathrm{keV}$ flux of Swift J1822.3-1606 even though it was detected in quiescence. The reasons for this are that Scholz et al. (2012) reported the lower bound for the $0.1-2.4 \mathrm{keV}$ flux to be zero (likely due to rounding since they do not claim their result is consistent with a non-detection) and that varying the spectral parameters within their reported uncertainties changed the estimated $2-10 \mathrm{keV}$ flux by over an order of magnitude. We therefore decided to report the highest such estimated flux as an upper limit. Additionally, Rea et al. (2012b) reported somewhat different spectral parameters for the same observation that gave a $2-10 \mathrm{keV}$ flux an order of magnitude greater than the one in the Table; it is this more conservative value that we use as an upper limit in calculations (including for the luminosity in Table 7) and Figures later in this paper. Finally, for the candidate magnetars AX J1818.8-1559 and AX J1845.0-0258, we provide separate spectral parameters and fluxes for single power-law and single blackbody models, but these results are for unconfirmed quiescent X-ray counterparts that may not be correctly identified.

The parameters in Table 3 are identical to what is provided in the main Table of our online catalog. However, online we also provide a table of alternative values including model parameter results from other observations (e.g. from different telescopes) which may also be of interest.

\subsection{Table 4: Optical and Near-Infrared Counterparts}

In Table 4 we summarize measurements of catalog magnetars made in the optical and near-infrared bands. Because magnetars are typically variable sources at these wavelengths, we list the range of magnitudes over which they have been detected in the $K_{s}, H, J, I, R, V, B$, and $U$ bands. We also provide the limiting magnitudes (usually $3 \sigma$ upper limits, but occasionally 2 or $5 \sigma$ ) in cases where observations failed to detect them.

As this Table provides merely a range of values, we reference only the detections with the lowest and highest reported magnitudes and/or the non-detection with the highest reported limiting magnitude. In cases where the same observation was analyzed in both non-refereed and refereed publications, we considered only the latter for inclusion. Finally, we must caution that any 'nonstandard' filter (that is, any filter other than the eight listed above, such as $K, K^{\prime}, z^{\prime}$, an $H S T$ filter, etc.) was assumed to be identical to whichever standard filter it most closely approximated, with no effort made to properly convert the magnitude. Therefore, please check the original references or the online catalog to confirm the filter used.

Seven magnetars have confirmed counterparts in the optical or near-infrared: $4 \mathrm{U} 0142+61$, SGR $0501+4516$, 1E 1048.1-5937, 1E 1547.0-5408, SGR 1806-20, XTE $\mathrm{J} 1810-197$, and $1 \mathrm{E} 2259+586$. Of these, optical pulsations have been detected from $4 \mathrm{U} 0142+61,1 \mathrm{E}$ 1048.1-5937, and SGR 0501+4516, of which the latter also shows good evidence for pulsations in the near-infrared band. There are also suggested counterparts for CXOU J010043.1-721134, 1E 1841-045, and SGR $1900+14$, but these are unconfirmed. There was a near-infrared counterpart proposed for 1RXS J170849.0-400910, but Testa et al. (2008) disputed the association when they found multiple fainter sources 
within the error circle of its X-ray position. To denote this ambiguity, we report the detected magnitude of the originally proposed candidate (Star 3 in Testa et al. 2008) as an upper limit marked with an asterisk. Similarly, $K_{s}$-band observations of SGR 1627-41 reveal multiple sources that may be the counterpart, so we list as an upper limit the detected magnitude of the brightest one (Source C in de Ugarte Postigo et al. 2009).

For more information, the online version of this catalog contains a more comprehensive table of optical and near-infrared counterparts. It tabulates individual observations of each magnetar, listing the date of observation, the detected (or limiting) magnitude, and any non-standard filters that were used.

\subsection{Table 5: Radio and Mid-Infrared Observations}

Table 5 contains information regarding radio and midinfrared observations of catalogued magnetars. For radio observations, we list all radio frequency ranges in which detections of pulsations have been reported, as well as the reported dispersion measure (DM). We also list, where available, the range of detected flux densities in the 1.4 and $2.0 \mathrm{GHz}$ bands; for sources that have never been detected at these wavelengths we provide an upper limit. Note that transient radio counterparts of SGRs $1806-20$ and $1900+14$ were detected following giant flares (Cameron et al. 2005; Frail et al. 1999), but since no pulsations were ever detected they are not included in this Table.

For mid-infrared observations, we list the reported fluxes or flux upper limits for catalogued magnetars at three wavelengths: $4.5 \mu \mathrm{m}, 8.0 \mu \mathrm{m}$, and $24 \mu \mathrm{m}$. Note that we are only concerned with the flux of the point source, so phenomena such as the infrared ring seen around SGR 1900+14 (Wachter et al. 2008) are not included.

\subsection{Table 6: Hard X-ray and Gamma-Ray Observations}

This Table contains the spectral properties of catalog magnetars in the hard $(>10 \mathrm{keV}) \mathrm{X}$-ray and gamma-ray range. The persistent hard X-ray emission from magnetars can typically be characterized by a power law, so we report the photon index $\Gamma$ and the unabsorbed 20 $150 \mathrm{keV}$ flux (estimated using WebPIMMS if flux was given for a different energy range) for both the pulsed and total emission, as denoted, respectively, by superscripts $p$ and $t$. Additionally, because the hard X-ray spectrum is expected to break or turn over at some point we also list the cut-off energy $E_{\text {cut }}$, although except for the case of $4 \mathrm{U} 0142+61$, only lower limits are available.

Most of the hard X-ray data in this Table comes from the INTEGRAL and Suzaku telescopes, and we generally tried to include results from both instruments (in that order) for each source where available. For results from INTEGRAL, we preferred the parameters derived using the longest integration time, though if it was clear that the parameters differed between two different time spans we included both results. As well, in cases where one publication gave multiple parameters for the same Suzaku observation, we chose the one preferred by the authors. Apart from those two telescopes, RXTE data was used for the pulsed emission from some sources, and the results for SGR J1745-2900 were found with NuSTAR.
Italicized values in the Table, seen for SGR $0501+4516$, 1E 1547.0-5408, and SGR J1745-2900, were taken when the source was in outburst, and here multiple values of the photon index and flux represent the source fading back into quiescence. Finally, we must clarify that the inconsistency seen for 1E 2259+586, where the pulsed flux is three times higher than the upper limit for the total flux, is due to pulsed emission only being seen by $R X T E$ up to $\sim 25 \mathrm{keV}$, meaning the extrapolated flux value reported in the Table must be greatly over-estimated.

Unlike at lower energies, no magnetars have yet been detected in gamma rays. We therefore provide only upper limits on their $0.1-10 \mathrm{Gev}$ flux, taken from Table 1 of Abdo et al. (2010).

\subsection{Table 7: Associations and Distances}

In Table 7 we tabulate distances to catalogued magnetars and related information. In particular, for each source we list any objects (e.g. supernova remnants, star clusters, etc.) that are proposed as associated with it, the age of the supernova remnant (where applicable and available), the distance measurement, and specifically to which object the distance is measured (be it the magnetar itself or an associated object). Associations whose validity has been disputed are noted. We also tabulate two parameters calculated using the distance $d$ : the height above the Galactic plane $z$, defined as $z=d \sin (b)$ where $b$ is the Galactic latitude (see Table 11); and the quiescent $2-10 \mathrm{keV}$ X-ray luminosity $L_{\mathrm{X}}$, defined as $L_{\mathrm{X}}=4 \pi d^{2} F_{\mathrm{X}}$ where $F_{\mathrm{X}}$ is the unabsorbed 2$10 \mathrm{keV}$ flux (see Table 3). For sources with no distance measurements, these derived parameters were estimated assuming a distance of $10 \mathrm{kpc}$. Additionally, since CXOU J010043.1-721134 and SGR 0526-66 are extragalactic magnetars located in the Magellanic Clouds, we do not calculate $z$ for them.

In cases where multiple distances to the same source exist in the literature, we chose the most recently measured value. Usually this distance was either consistent with earlier measurements or generally accepted over them among the literature, but for 1E 1048.1-5937 and $1 \mathrm{E} 2259+586$ there is some disagreement in the literature between multiple incompatible distance measurements. For more details, see the table of alternate values in our online catalog which lists these other distance measurements with references, or see the discussion in the papers cited in Table 7.

\section{DISCUSSION}

Figure 1 shows the accumulated number of known confirmed magnetars as a function of year up to the present day. The vertical dashed line shows the launch date of Swift with its Burst Alert Telescope (BAT) onboard (Barthelmy et al. 2005), and the dot-dashed line shows the launch date of the Fermi mission and its Gammaray Burst Monitor (GBM; Meegan et al. 2009). It is no coincidence that the slope of the accumulation increases significantly when BAT became active and again when GBM turned on, since they are extremely well designed to detect bright magnetar bursts. In fact they, and previous all-sky X-ray/soft-gamma ray monitors, were designed to detect gamma-ray bursts, which are one-time bursters of cosmological origin. Hence these monitors 


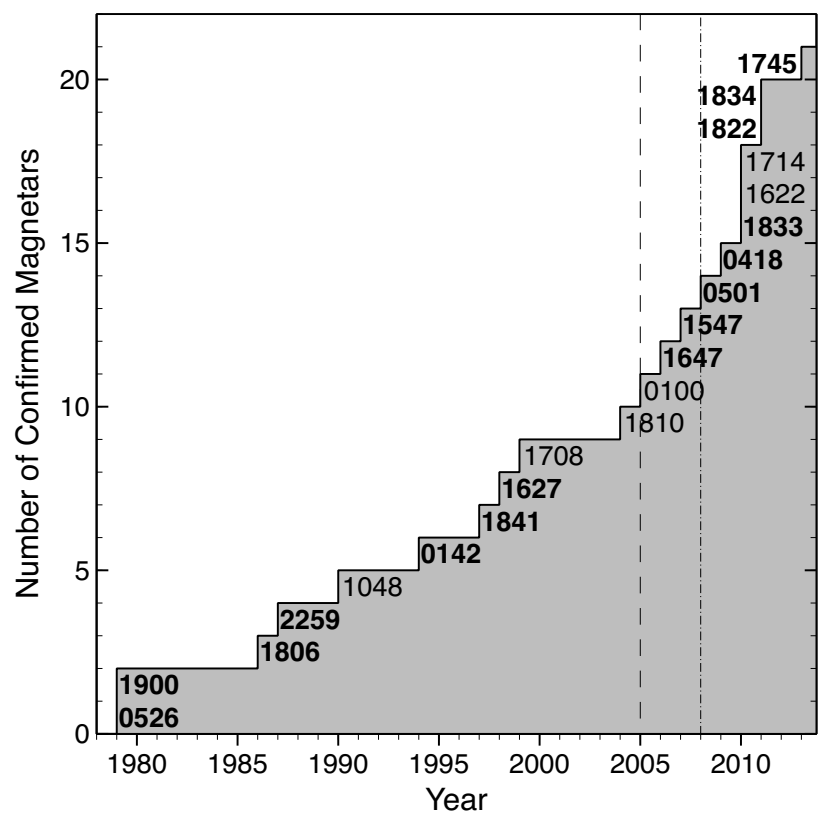

Figure 1. Number of confirmed magnetars discovered over time. Labels in boldface indicate the source was either discovered or later detected by an all-sky X-ray/soft gamma-ray burst monitor. The dashed and dot-dashed lines mark the launches of Swift in 2005 and Fermi in 2008, respectively.

are specifically designed to view the entire sky in an unbiased fashion, and so are sensitive to Galactic, repeating bursters regardless of location in the Galaxy. Thus they have yielded a directionally unbiased sample of magnetars, selected only for their magnetar activity, namely bursting. In Figure 1, sources which were either discovered by an all-sky X-ray/soft gamma-ray monitor, or which were later detected (and therefore could have been discovered) by one, are highlighted in italics, for this reason.

Many known magnetars have thus been found via their bursting behavior, which raises an important point regarding how they are named. Because burst monitors have tended to find them, magnetars have often been named with the designation "SGR" in recent years (see Tables). We argue strongly that this naming convention requires amendment because as discussed in this work and extensively elsewhere (e.g. Gavriil et al. 2002; Kaspi et al. 2003; Woods \& Thompson 2006; Mereghetti 2008; Kaspi 2010; Mereghetti 2013; Rea \& Esposito 2011) the distinction between sources designated as "AXP" and "SGRs" has been largely erased via the discovery of objects which have properties previously ascribed to both categories. It is today very hard to classify some sources as one or the other; rather it has become clear that there is a continuous spectrum of magnetar-type activity which can even include some high- $B$ rotation-powered pulsars (e.g. PSR J1846-0258; Gavriil et al. 2008). Sources discovered via bursting seem like an SGR but may later lie dormant and burstless for decades and seem like an AXP (e.g. SGR 0526-66; Kulkarni et al. 2003). Meanwhile sources discovered in quiescence and showing no bursts, therefore initially classified as AXPs, may later begin bursting (e.g. 1E 1547.0-5408; Gelfand \& Gaensler 2007; Israel et al.
2010; Kaneko et al. 2010). A source's fixed designation can clearly not depend on behavior that is constantly evolving. We instead propose a naming scheme that designates magnetars by the acronym ' $\mathrm{MG}$,' analogous to 'PSR' as used for pulsars. A list of MG names is provided in Table 8 in the Appendix. Another possibility would be to keep names as with other X-ray sources, for which the initial prefix is informative regarding the discovery telescope, as for, e.g. XTE J1810-197, discovered by $R X T E$. We suggest these, and other possible alternatives, be discussed seriously by the community.

\subsection{Spatial Properties}

Figure 2 shows a top-down view of the Galactic Plane with the Galactic Center at coordinate $(0,0)$. The greyscale is the distribution of free electrons from the model of Cordes \& Lazio (2002) and delineates the approximate locations of the spiral arms. Galactic disk radio pulsars from the ATNF catalog ${ }^{4}$ are denoted with blue dots. The so-called 'X-ray Isolated Neutron Stars' (XINSs; see Kaspi et al. 2006; Haberl 2007; Kaplan 2008 for reviews) are shown in yellow and are without exception very close to the Sun. The magnetars are shown as red circles, with their estimated distance uncertainties indicated. Note the magnetar SGR J1745-2900 whose location is consistent with the Galactic Center. This plot clearly indicates the preponderance of magnetars in the direction of the inner Galaxy, but with several notable exceptions in the outer Galaxy. The lack of clustering around the solar system of magnetars, particularly compared with the known radio pulsar population, suggests that fewer selection effects exist in the known magnetar population, apart from selection for bursting, particularly in the Swift and Fermi eras.

Figure 3 presents histograms of the distribution of ATNF Galactic radio pulsars and magnetars in Galactic longitude $l$. The radio pulsars are color-coded for age as indicated and the magnetars are indicated by the hatched red region. As surmised from Figure2, the known Galactic magnetars are more concentrated in the inner Galaxy, which is not a mere selection effect, again given the allsky nature of the burst detectors. While, again, selection effects in radio pulsar surveys may hinder the detection of the youngest objects in the very inner Galaxy where multipath scattering is important (Rickett 1990), we can nevertheless compare the $l$-distributions of the magnetars and young radio pulsars using a Kolmogorov-Smirnov (KS) test to see if they are consistent with having been drawn from the same distribution. For radio pulsars having $\tau<10 \mathrm{kyr}$, we find a KS probability of the null hypothesis of $p=0.14$, and likewise we also find $p=0.14$ for $\tau<100 \mathrm{kyr}$. Hence we cannot exclude that the two distributions are consistent with being drawn from the same underlying distribution.

Figure 4 presents histograms of the distribution of ATNF Galactic disk radio pulsars and magnetars in Galactic latitude $b$ in degrees, with a zoom-in to the most populated region to better highlight the magnetars which are relatively few in number. Note that with the exception of just one magnetar (SGR 0418+5729, but see $\S 3.2$ ), all known Galactic magnetars lie within $2^{\circ}$ of the

4 http://www.atnf.csiro.au/research/pulsar/psrcat/, version 1.47 


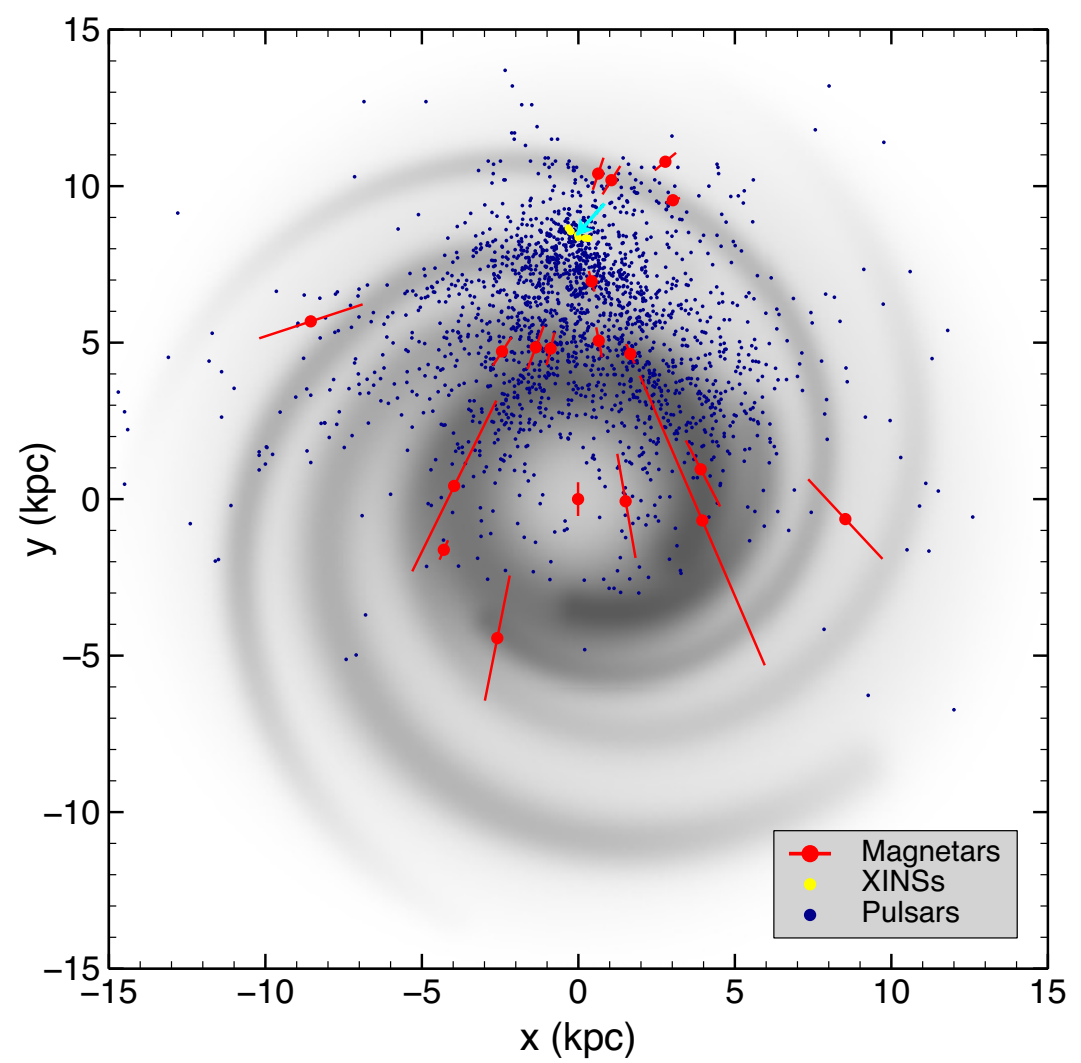

Figure 2. Top-down view of the Galaxy, with the Galactic Center at coordinates $(0,0)$ and the location of the Sun marked by a cyan arrow at coordinates $(0,8.5)$. The grayscale shows the distribution of free electrons given by the model of Cordes \& Lazio (2002). The magnetars are denoted by red circles with distance uncertainties indicated by the lines, the X-ray Isolated Neutron Stars (XINSs) are shown by the yellow circles near the Sun, and the locations of all other pulsars are given by the blue dots.
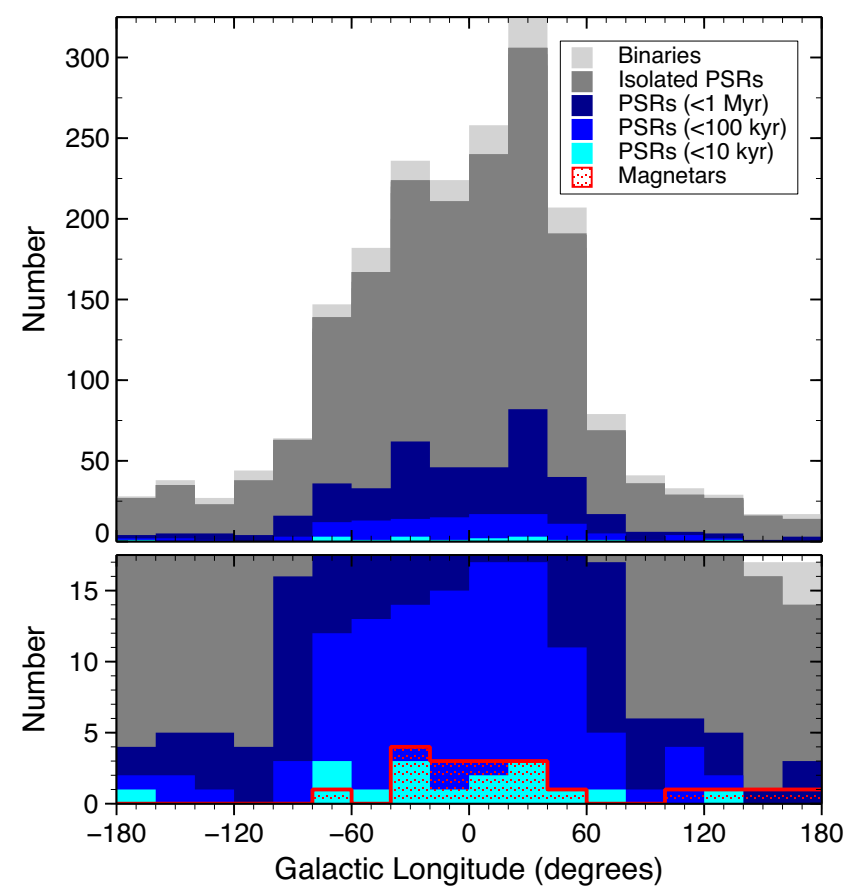

Figure 3. Top panel: Distribution in Galactic longitude $l$ of all Galactic disc pulsars. Young, isolated pulsars are indicated by the various blue regions ( $<10 \mathrm{kyr}$ : cyan; $<100 \mathrm{kyr}$ : blue; and $<1 \mathrm{Myr}$ : dark blue), with the remaining isolated pulsars and pulsars in binary systems shown respectively by the grey and light grey regions. Bottom panel: Zoom-in to better show the distribution of the magnetars, given by the hatched red region, and the youngest pulsars.

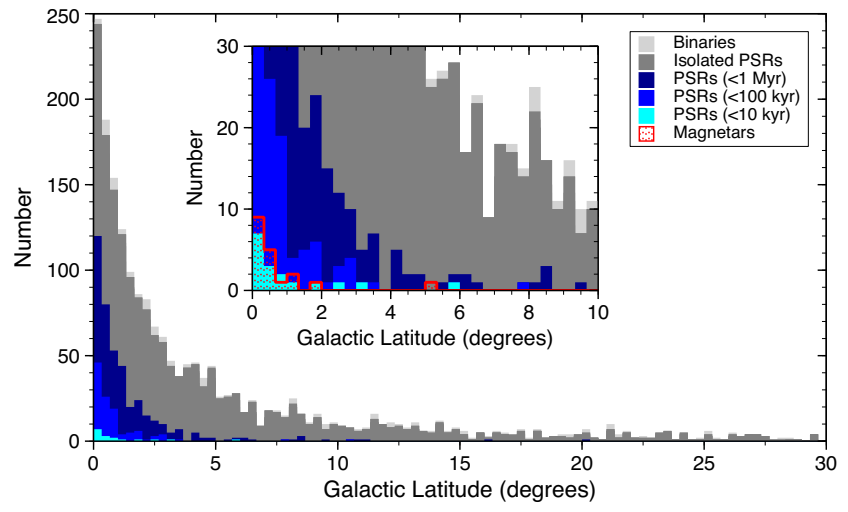

Figure 4. Distribution in Galactic latitude $b$ of all Galactic disc pulsars (colors as in Figure 3). Inset: Zoom in near the origin with the magnetars shown by the hatched red region.

Galactic Plane, consistent with their interpretation as a population of young objects. The physical scale height in $\mathrm{pc}$, however, is more relevant in understanding the Galactic distribution, which we discuss below.

\subsubsection{Magnetar Scale Height}

In Figure 5 (bottom panel) we plot a histogram of the distribution of magnetars as a function of their height above the Galactic Plane $z \equiv d \sin (b)$ in pc, where $d$ is the distance to the object in pc. It is evident that the distribution does not peak at $z=0$, meaning that simply fitting the distribution to $\exp (-|z| / h)$ as is typically done for pulsars will not give an accurate result. 


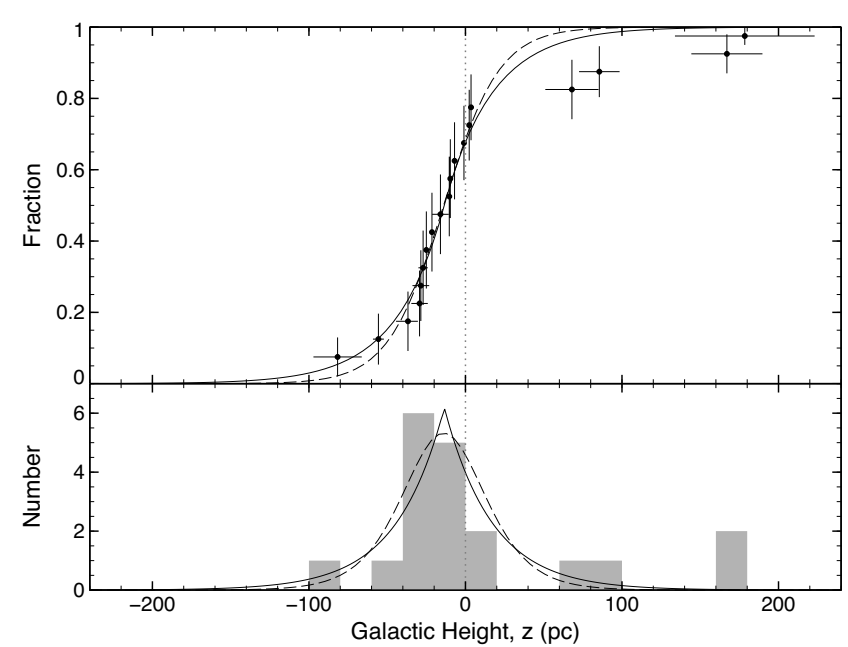

Figure 5. Top panel: Cumulative distribution function of the height $z$ above the Galactic plane for the 19 magnetars located in the Milky Way. Data are fit to an exponential model (solid line) and a self-gravitating, isothermal disc model (dashed line). See text for details. Bottom panel: Histogram of the distribution in $z$ of the Galactic magnetars. Lines are as above.

The Sun does not lie in the Galactic plane as defined by the magnetars. We therefore used two models that included a term for the height of the Sun: an exponential model and a self-gravitating, isothermal disc model (e.g. Bahcall 1984):

$n(z)=n_{0} \exp \left(-\frac{\left|z+z_{0}\right|}{h_{e}}\right), n(z)=n_{0} \operatorname{sech}^{2}\left(\frac{\left|z+z_{0}\right|}{2 h_{s}}\right)$,

where $h_{e}$ and $h_{s}$ are, respectively, the scale heights for the exponential and self-gravitating models, and $z_{0}$ is the height of the Sun above the Galactic plane.

Because of the small number of sources we can work with, as well as the significant distance uncertainties involved, we constructed and fit our models to the unbinned cumulative distribution function (top panel of Figure 5) rather than fitting to the histogram directly. The resulting best-fit values were $h_{e}=30.7 \pm 5.9 \mathrm{pc}$ and $z_{0}=13.5 \pm 2.6 \mathrm{pc}$ for the exponential model and $h_{s}=17.9 \pm 3.3 \mathrm{pc}$ and $z_{0}=13.9 \pm 2.5 \mathrm{pc}$ for the selfgravitating model. Note that the listed $1 \sigma$ uncertainties include both the statistical uncertainty from fitting as well as the $1 \sigma$ uncertainty obtained from a Monte Carlo analysis in which we randomly varied the distance (and therefore $z$ ) to each magnetar within their uncertainties. In an effort to check the stability of our results, we also repeated this procedure for a few different subsets of the magnetar population. In particular, we tried fitting the two models to only the 14 Galactic magnetars that have been detected by all-sky monitors (see Figure 11) since those sources do not have any sort of directional selection effects. As well, since the bottom panel of Figure 5 suggests that fitting to the cumulative distribution weights the outlying points more heavily than fitting to the histogram would, we also tested fits excluding the two sources with $|z|>100$ pc (SGR $0418+5729$ and SGR $1900+14)$. We found that these changes tended to decrease $h_{e}$ and $h_{s}$ and increase $z_{0}$; overall the best-fit values for the scale height varied in the range $\sim 20-31 \mathrm{pc}$ for $h_{e}$ and $\sim 13-18 \mathrm{pc}$ for $h_{s}$, and the best-fit values for the height of the Sun $z_{0}$ ranged from $\sim 13-22 \mathrm{pc}$ for both models.

For comparison, we repeated the same procedure for all ATNF pulsars with characteristic age less than $100 \mathrm{kyr}$ (excluding magnetars) and found scale heights $h_{e}=$ $61 \pm 5 \mathrm{pc}$ and $h_{s}=39 \pm 3 \mathrm{pc}$, approximately twice as large as our results for the magnetars. However, note that unlike the magnetars, strong selection effects are at work in shaping the known population of radio pulsars; see, e.g. Faucher-Giguère \& Kaspi (2006) for a detailed discussion. Indeed, it is generally more difficult to find faster - hence typically younger - radio pulsars closer to the Galactic Plane because of the deleterious effects of dispersion smearing and scattering, though recent pulsar surveys of the radio sky are improving the situation (Manchester et al. 2001; Lazarus 2013). Hence, we may easily have over-estimated the scale height of young radio pulsars. Regardless, it is unsurprising that the scale height of magnetars is smaller or similar to that of young radio pulsars, given that magnetars are believed to be young neutron stars.

We can also compare our results with measurements in the literature of the scale height of OB stars, the progenitors of neutron stars. In particular, Reed (2000) and Elias et al. (2006) derived values of $h_{e}(45 \pm 20 \mathrm{pc}$ and $34 \pm 2 \mathrm{pc}$, respectively) which overlap with the upper end of our own range, but other measurements by Joshi (2007) $\left(h_{e}=61.4 \pm 2.6 \mathrm{pc}\right)$ and Maíz-Apellániz (2001) $\left(h_{s}=34.2 \mathrm{pc}\right)$ are significantly greater. This discrepancy could argue in favor of the hypothesis that magnetars are born from massive progenitors (Figer et al. 2005; Muno et al. 2006) if the OB star scale height depends on stellar mass such that more massive $\mathrm{O}$ stars have a scale height that agrees with that of the magnetars. Unfortunately, there is no compelling evidence for such a dependence on stellar mass via spectral type (Maíz Apellániz et al. 2008), although it cannot yet be said to be disproven either. Nevertheless, we argue that the observed magnetar scale height favors massive progenitors. In particular, $9 M_{\odot}$ stars have an expected lifetime of about $20 \mathrm{Myr}$ (Milhalas \& Binney 1981), so assuming a peculiar velocity of $\sim 5-10 \mathrm{~km} \mathrm{~s}^{-1}$ (Gies 1987) they will have travelled $\sim 70-140 \mathrm{pc}$ in the direction perpendicular to the Plane by the end of their lives, significantly greater than the $\sim 20-30 \mathrm{pc}$ magnetar scale height. Conversely, $40 M_{\odot}$ stars live for approximately $1 \mathrm{Myr}$, so given the same velocity they will travel only $\sim 3-7 \mathrm{pc}$ during their life span, a much smaller value that is consistent with the observed distribution of magnetars.

Finally, we find that our measurement of the height of the Sun above the Galactic plane $z_{0}$ agrees well with previous measurements, which generally all fall within the range of 10-30 pc (e.g. 10-12 pc, Reed 1997; $15 \pm$ $3 \mathrm{pc}$, Conti \& Vacca 1990; $16 \pm 5$ pc, Elias et al. 2006; $24.2 \pm 2.1$ pc, Maíz-Apellániz 2001).

\subsection{Timing Properties}

In Figures 6 6] we show histograms of pulse periods and properties inferred from timing for the radio pulsar population, the XINS, and the magnetars. Figure 6 shows the periods, and it is clear that magnetars have longer spin periods than the vast majority of the radio pulsars, although there is overlap with the long-period tail of the radio pulsar distribution. Additionally, the spin periods of the magnetars are very similar to those 


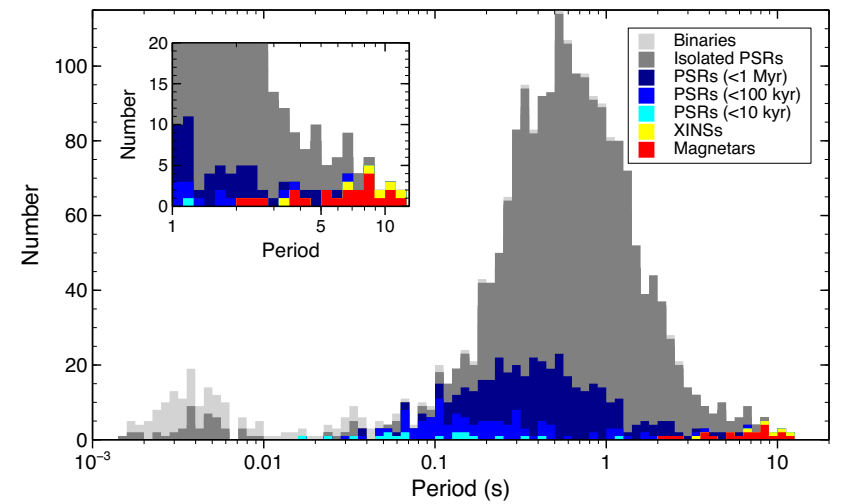

Figure 6. Histogram showing the distribution in pulse period of all known radio pulsars (colors as in Figure 3), XINSs (yellow), and magnetars (red). Inset: Zoom in on $P>1 \mathrm{~s}$, where the magnetars are all located.

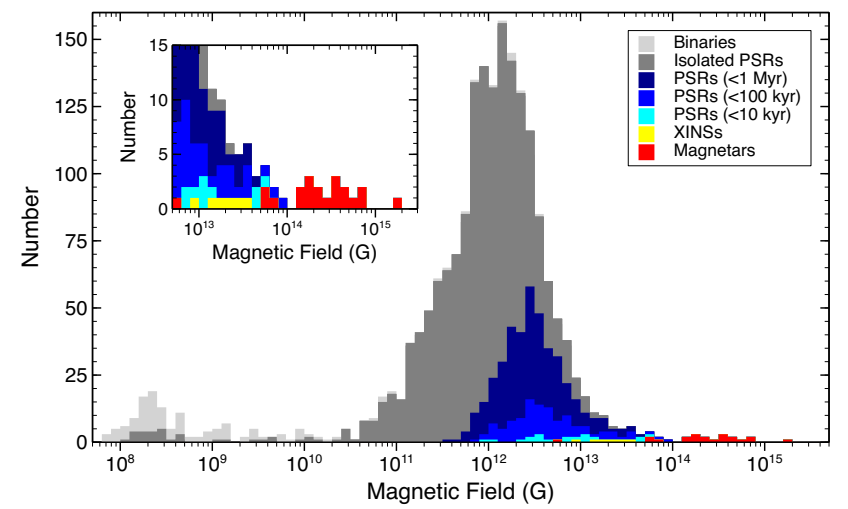

Figure 7. Histogram showing the distribution in magnetic field $B$ of all known radio pulsars, XINSs, and magnetars for which $\dot{P}$ has been measured (colors as in Figure 6). Inset: Zoom in on $B>5 \times 10^{12} \mathrm{G}$ to better show the distribution of the magnetars.

of the XINSs. Indeed models of magnetic and thermal evolution in neutron stars are suggestive of an evolutionary relationship between magnetars and XINSs, with the latter descendants of the former (Vigano et al. 2013; Popov et al. 2010). Notable also is the small range of magnetar periods, especially compared with those of radio pulsars. The paucity at shorter periods is understood as being a result of their rapid spin-down due to their high $B$ fields. On the other hand, the reason for the lack of magnetars spin periods longer than $12 \mathrm{~s}$ is not well established; one possibility is that by the time objects reach so long a period, their fields have decayed so much that the hallmark activity and X-ray emission has ceased (e.g. Colpi et al. 2000). On the other hand, the longest period magnetar yet known (1E 1841-045) also has the highest persistent $2-10 \mathrm{keV}$ luminosity (Table 3). This suggests that even longer-period magnetars are yet to be found.

In Figure 7 distributions of the spin-inferred surface dipolar magnetic field $B$ are shown. Again it is clear that the typical magnetar field is $2-3$ orders of magnitude greater than that of the typical radio pulsar, and indeed the overlap of the magnetar field distribution with the high- $B$ tail of the radio pulsar distribution is relatively small, restricted to three objects (SGR 0418+5729, Swift J1822.3-1606 and 1E 2259+586). Indeed the magnetars largely stand alone on this plot, with the XINSs hav-

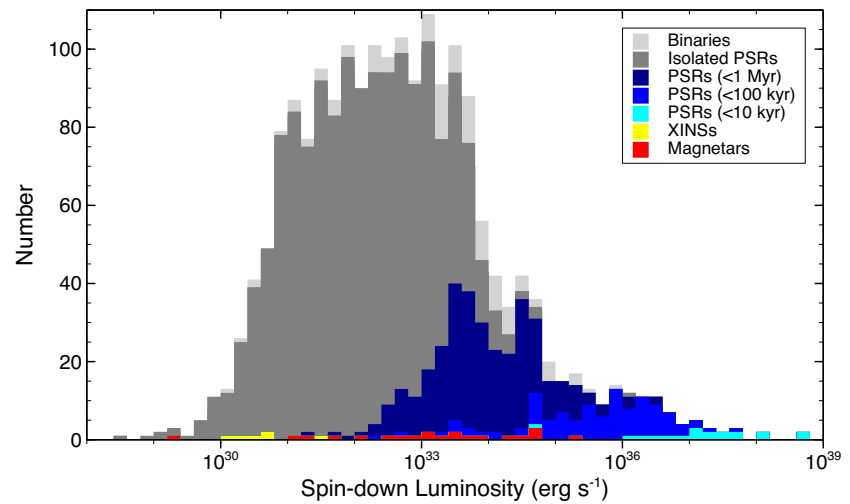

Figure 8. Same as Figure 7 but for the spin-down luminosity $\dot{E}$.

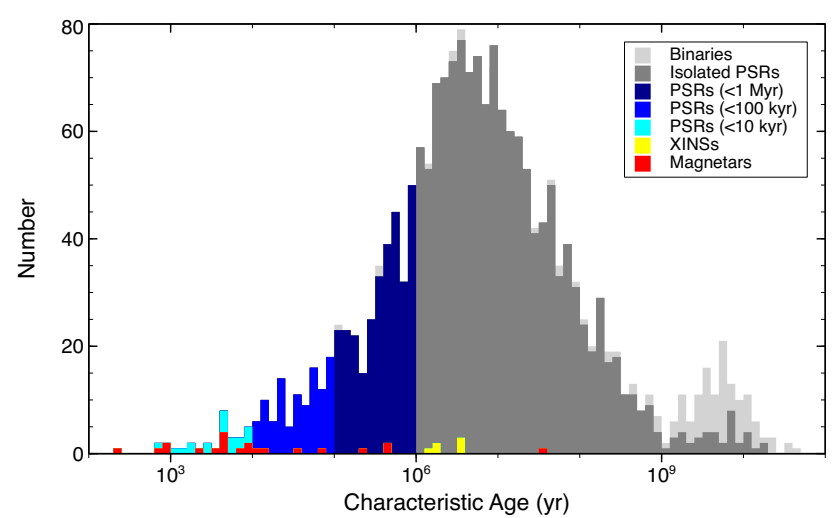

Figure 9. Same as Figure 7 but for the characteristic age.

ing intermediate field values. Much has been made of the discovery of SGR 0418+5729 (Rea et al. 2010) given its low spin-inferred $B$ strength, however Figure 7 makes clear that when viewing the overall known magnetar population, which is largely selected in an unbiased fashion based on burst activity, low- $B$ objects are the exception.

Interestingly, this Figure also shows that the younger known radio pulsars tend to have $B$ fields higher than the field of the typical known radio pulsar. This might naively suggest that radio pulsar magnetic fields decay with time. On the other hand, higher-field sources spin down more rapidly, reaching the death line sooner, so the most common radio pulsar found is likelier to have lower $B$ since it has a longer lifetime. The small scale height for magnetars described in 3.1.1 then is consistent with the relative numbers of high- $B$ and low- $B$ magnetars: the objects with the highest fields have the smallest lifetimes hence have little time to leave their birthplace. Indeed it is unsurprising that the source with the lowest known $B$ field, SGR J0418+5729, is also the magnetar furthest from the Galactic Plane (see Table 7 ).

Figure 8 shows a histogram of the spin-down luminosity $\dot{E}$. In this plot, the magnetars are distributed fairly uniformly but broadly, spanning a full five orders of magnitude. Below we consider correlations between $\dot{E}$ and radiative properties, but for the moment we note that the broad range of $\dot{E}$ - in contrast to the far narrower and more distinctive range in $B$ - suggests that the former does not play a dominant role in the high-energy emission from magnetars.

Figure 9 shows distributions of characteristic age $\tau_{c}$. 
As with $\dot{E}$, magnetar ages are uniformly but broadly distributed. The breadth is interestingly at odds with their very small Galactic scale height (\$3.1.1), even given magnetars' relatively low mean velocity (Tendulkar et al. 2013). This indicates that the characteristic ages of magnetars are poor proxies for their true ages. Independent evidence for this is already clear from the disparity in the characteristic age of $1 \mathrm{E} 2259+586$ (230 kyr; see Table 2) compared with the estimated age of its host supernova remnant CTB 109 (14 kyr; see Table 7). Note though that the latter example is extreme; in contrast stands $1 \mathrm{E} 1841-045$ whose characteristic age, $4.8 \mathrm{kyr}$, is much closer (though still larger) than the estimated age of its host remnant, Kes $73(0.5-1 \mathrm{kyr})$. The primary reason for the breadth in characteristic age is unclear. In some cases it may be at least partially due to fluctuations in $\dot{P}$ (as in 1E 1048.1-5937; Gavriil \& Kaspi 2004, Dib and Kaspi 2013) which could bias a short-term measurement. Alternatively, torque decay as the magnetic field decays is also a likely factor (e.g. Thompson et al. 2002).

In Figure 10 we present a $P-\dot{P}$ diagram which includes all catalogued magnetars, XINSs, and radio pulsars having measured $P$ and $\dot{P}$. This presentation re-emphasizes the relatively long periods and large spin-down rates of the magnetar population. Also made clear by this diagram is the overlap in $P-\dot{P}$ space between magnetars and some radio pulsars. This is suggestive of potential magnetar activity from these apparently high- $B$ radio pulsars. The observed short-lived magnetar activity from rotation-powered pulsar PSR J1846-0258 supports this idea (Gavriil et al. 2008), as does apparently enhanced thermal X-ray emission from high- $B$ radio pulsars compared with that from lower- $B$ radio pulsars of comparable age (Kaspi \& McLaughlin 2005; Olausen et al. 2010; Zhu et al. 2011; Olausen et al. 2013). Figure 10 also makes clearer that XINS spin properties do not fully overlap with those of magnetars; the former have smaller spin-down rates hence smaller inferred $B$. These objects are thus evidence for torque decay in high- $B$ neutron stars and suggest XINS could be descendants of magnetars as mentioned above.

\subsection{X-ray Properties}

Figure 11 plots photon index $\Gamma$ and blackbody temperature $k T$ versus spin-inferred magnetic field $B$ for those sources which have a power-law or blackbody component in their quiescent X-ray spectrum (see Table 3). The left graph shows evidence of a trend where $\Gamma$ decreases as $B$ increases, previously identified in Kaspi \& Boydstun (2010) and in a different but analogous form by Enoto et al. (2010a). Following the example of Kaspi \& Bovdstun we attempt to quantify the trend by calculating Pearson's correlation coefficient, finding $r=-0.79$ (upper limits were included in the calculation of $r$ by assuming a value of half of the upper limit). For a sample size of $N=11$, this result gives a (two-tailed) probability for the null hypothesis of $p=0.0035$, slightly higher than the result obtained by Kaspi \& Boydstun but still near the $3 \sigma$ level. Conversely, examination of the plot on the right for evidence of a correlation between $k T$ and $B$ revealed none; in particular we obtained $r=0.36$ for $N=15$, giving $p=0.18$ which does not exclude the null hypothe- sis. Overall, these results support the "twisted magnetosphere" model of Thompson et al. (2002), further developed by Beloborodov (2009), which predicts that a higher $B$ field drives stronger currents in the star's magnetosphere which in turn produces brighter and harder non-thermal X-ray emission.

In Figure 12 we plot $L_{\mathrm{X}}$, the quiescent X-ray luminosity in the $2-10 \mathrm{keV}$ energy band, against $\Gamma$ and $k T$ for the same sources as above. We again calculate the correlation coefficient $r$ but in both cases we derive a null-hypothesis probability of $0.02-0.03$, not low enough to comfortably reject. Certainly a correlation between $L_{\mathrm{X}}$ and $k T$ is not evident; notice how the luminosity spans five orders of magnitude at $k T \approx 0.3 \mathrm{keV}$. Likewise, $L_{\mathrm{X}}$ spans more than two orders of magnitude at $\Gamma \approx 3.8$. On the other hand, there does appear to be an excluded region in the $L_{\mathrm{X}}$ vs. $\Gamma$ graph where one would find lower-luminosity sources with hard power laws (although given the large uncertainty in $\Gamma$, SGR 1627-41 cannot be excluded from encroaching into this region). This cannot simply be due to a selection effect, because given the same luminosity a harder source will produce less flux at energies prone to Galactic absorption than a softer one and should therefore be easier to detect. As indicated above, a harder spectrum is associated with greater X-ray luminosity in the twisted magnetosphere model, so such a gap is consistent with that. However, the model also implies that we should not expect to see high-luminosity sources with soft power laws. We do note that a calculation of $r$ excluding the upper-rightmost point (4U 0142+61) drops the probability of the null hypothesis below $1 \%(r=-0.80$ for $N=10, p=0.0054)$, although there is no compelling reason to ignore or discard it.

In the leftmost panel of Figure 13 we show the quiescent $2-10 \mathrm{keV}$ luminosity $L_{\mathrm{X}}$ as a function of $B$. This plot is an update of Figure 4 from An et al. (2012), although we do not assume the same uncertainties as that paper when drawing the error bars. The solid and open circles denote the magnetars and the open diamonds represent the five high- $B$ radio pulsars also considered by An et al. . A possible correlation can be seen in the data, so as above we investigated it by calculating Pearson's correlation coefficient and found that it strongly supports the existence of such a correlation $(r=0.72$ for $N=21$, $\left.p=2.2 \times 10^{-4}\right)$. We noticed, however, that there were points in the upper right and lower left corners of the graph, marked by the open circles, that could have had a significant impact on the calculation of $r$. Removal of these extreme points, SGRs $0418+5729$ and 1806-20, still resulted in rejection of the null hypothesis $(r=0.60$ for $N=19, p=0.0064$ ). Furthermore, as in An et al. the inclusion of high- $B$ radio pulsars only strengthened the relation $\left(r=0.73\right.$ for $\left.N=24, p=5 \times 10^{-5}\right)$, so it appears that there could be a genuine correlation between $L_{\mathrm{X}}$ and $B$ in high-magnetic-field neutron stars. There are two other magnetars, $4 \mathrm{U} 0142+61$ and $1 \mathrm{E}$ $2259+586$, that stand out in the plot with unusually high luminosities given their lower magnetic fields. This may suggest that their magnetic fields have strong nondipolar components, not seen in the spin-inferred field $B$, that would bring the total field strength in line with the other magnetars of similar $L_{\mathrm{X}}$. Overall, though, these results 


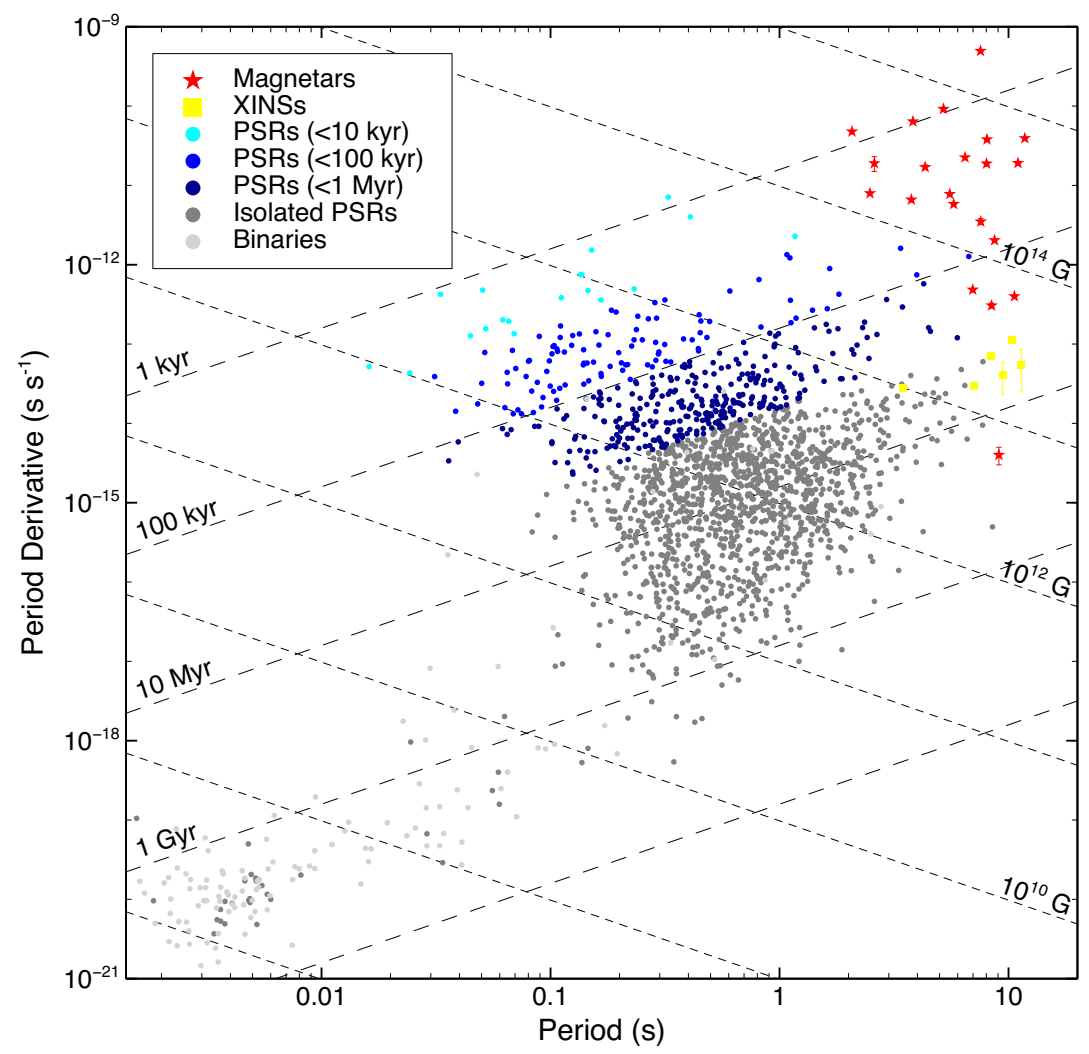

Figure 10. $P-\dot{P}$ diagram for all known radio pulsars (grey or blue dots as indicated), XINSs (yellow squares), and magnetars (red stars).
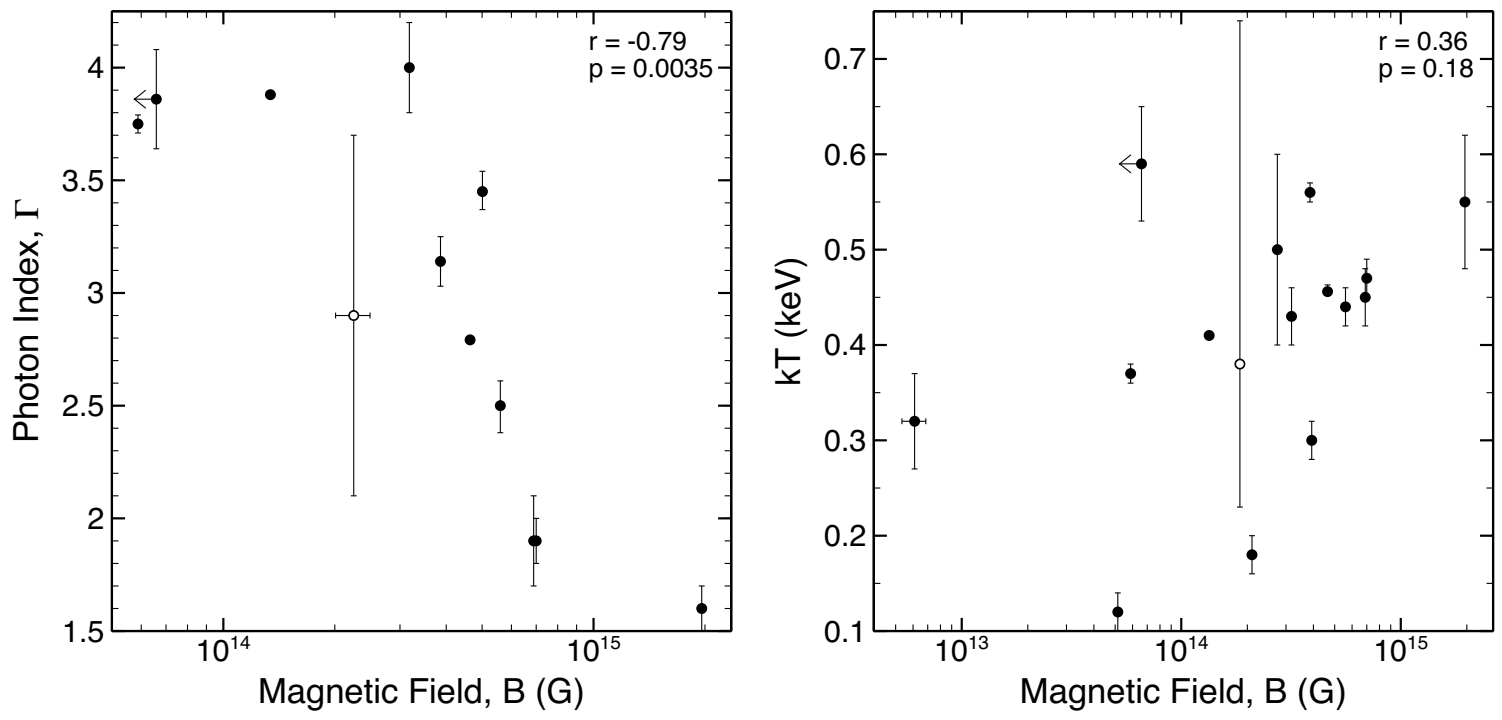

Figure 11. Photon index $\Gamma$ (left) and blackbody temperature $k T$ (right) versus magnetic field $B$. The correlation coefficient $r$ and associated null-hypothesis probability $p$ are shown in the upper right of each plot. The open circles represent points which were excluded from the calculation of $r$ due to their large uncertainties (SGR 1627-41 in $\Gamma$ and SGR 0501+4516 in $k T$ ).

support the idea that there is a continuum in the $\mathrm{X}$ ray luminosities of high- $B$ radio pulsars and magnetars (see An et al. 2012 for further discussion) as expected on physical grounds based on magnetic dissipation and expected magnetothermal evolution (Thompson \& Duncan 1996; Pons et al. 2009).

In the middle panel of Figure 13 we show a plot of $L_{\mathrm{X}}$ versus spin-down luminosity $\dot{E}$. The panel shows little more than a scatter plot, as borne out by the correlation coefficient $(r=0.38$ for $N=21, p=0.087 ; r=0.095$, $p=0.70$ with SGRs $0418+5729$ and $1806-20$ removed). This result is expected in the magnetar model, since the $\mathrm{X}$-ray emission is not powered by the rotational energy. The rightmost panel of Figure 13 presents $L_{\mathrm{X}}$ vs. characteristic age, and like the previous graph there is no visual sign of a strong trend. Naively calculating the correlation coefficient, however, does show evidence for a relation $(r=-0.56$ for $N=21, p=0.0078)$, but it is carried 

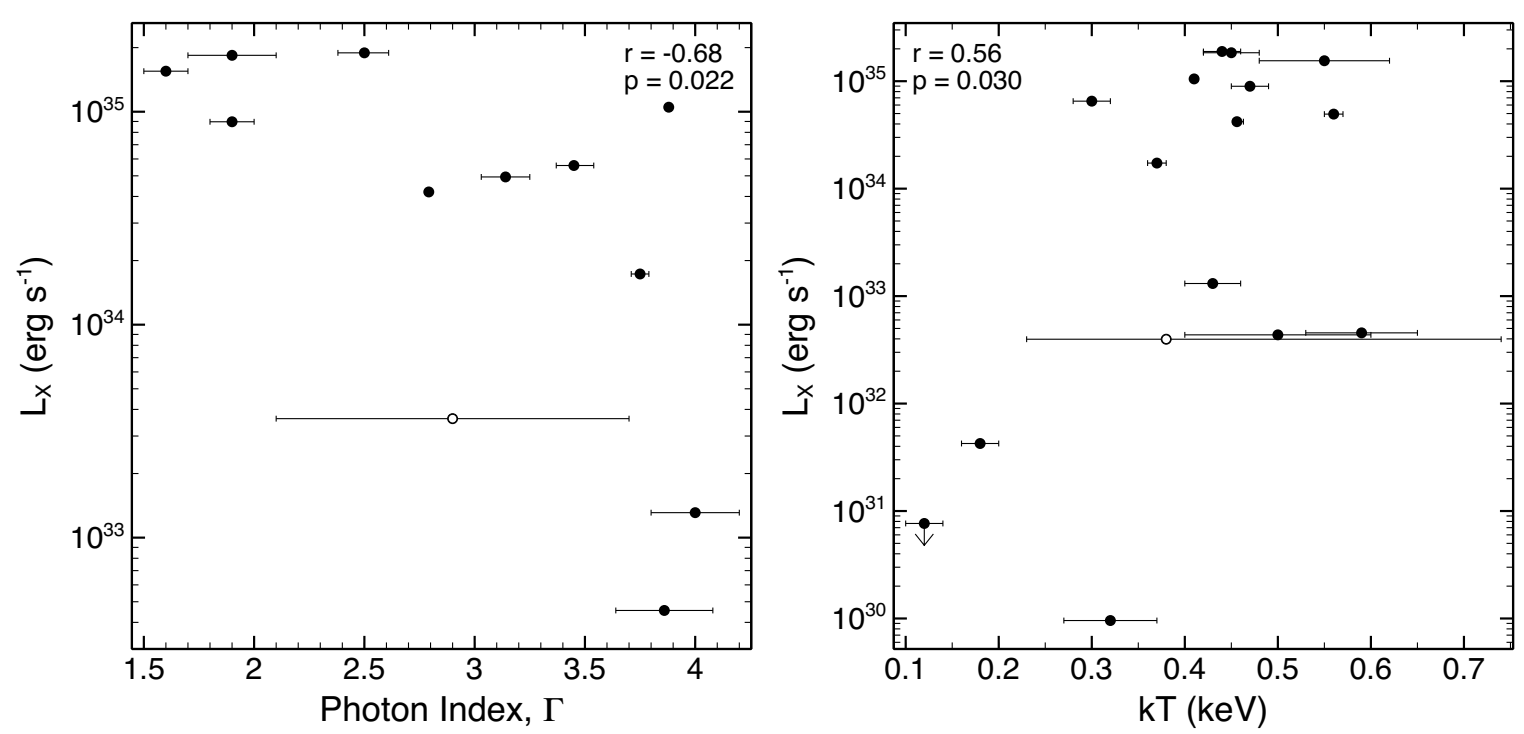

Figure 12. Quiescent $2-10 \mathrm{keV}$ X-ray luminosity $L_{\mathrm{X}}$ vs. $\Gamma$ (left) and $k T$ (right). The correlation coefficient $r$ and null-hypothesis probability $p$ are shown in the upper right or left of each plot, and the open circles are the same as in Figure 11

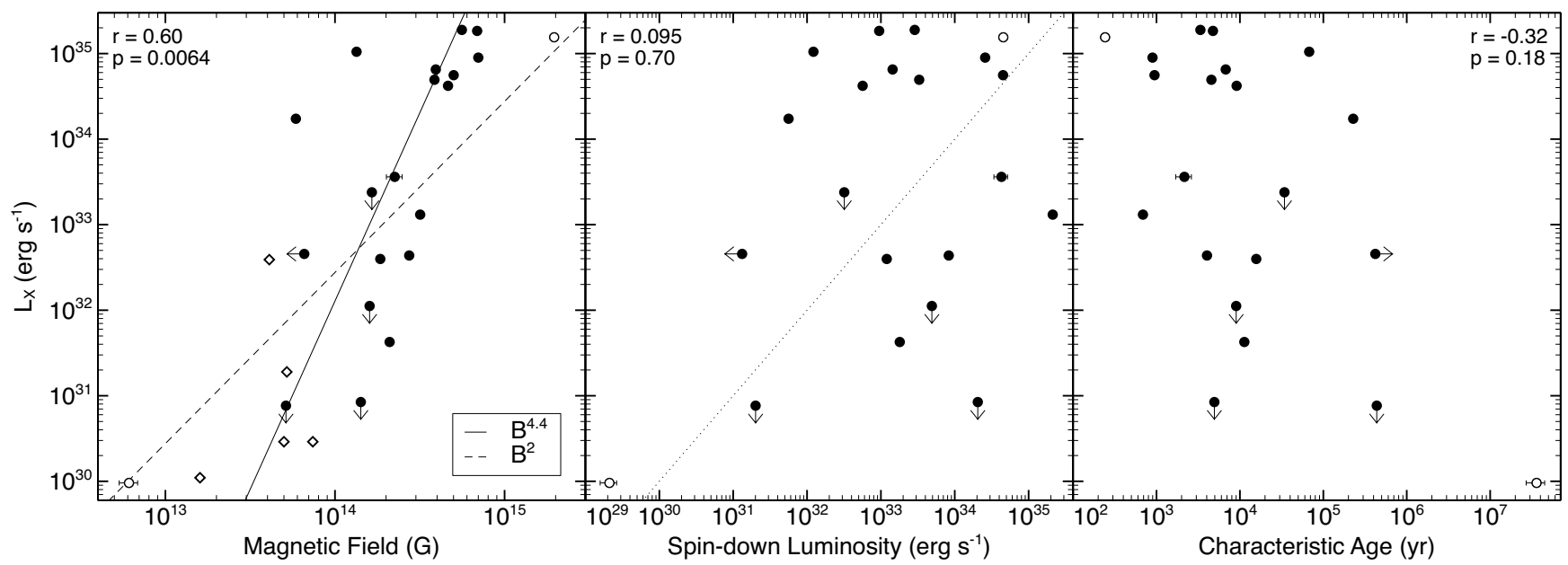

Figure 13. Left panel: Quiescent $2-10 \mathrm{keV}$ X-ray luminosity $L_{\mathrm{X}}$ vs. $B$ for the magnetars (solid and open circles) and select high- $B$ radio pulsars (open diamonds). Data for the radio pulsars was taken from Table 3 in An et al. (2012). The solid and dashed lines show fits to the data for the relations $L_{\mathrm{X}} \propto B^{4.4}$ and $L_{\mathrm{X}} \propto B^{2}$, respectively. Middle panel: $L_{\mathrm{X}}$ vs. $\dot{E}$. The dotted line marks $L_{\mathrm{X}} / \dot{E}=1$. Right panel: $L_{\mathrm{X}}$ vs. $\tau_{c}$. All panels: The open circles mark SGRs $0418+5729$ and $1806-20$. Because these two magnetars lie at opposite corners of each graph, they were excluded from the calculation of the correlation coefficient $r$, shown together with the null-hypothesis probability $p$ in the upper left or right of each plot, to ensure that a correlation did not depend on their presence.

entirely by SGRs $0418+5729$ and $1806-20(r=-0.32$, $p=0.18$ with those two points removed). Again, this is unsurprising because not only are the characteristic ages of magnetars not necessarily good measures of their true ages as discussed above, but the $2-10 \mathrm{keV}$ luminosity is dominated by the non-thermal emission so we do not expect to detect a cooling trend anyway.

Figure 14 shows a plot of $k T$ versus characteristic age for magnetars, XINSs, and select radio pulsars, with high- $B\left(\geq 10^{13} \mathrm{G}\right)$ sources shown in red and yellow (with the exception that the low-field magnetar, SGR $0418+5729$, is also shown in yellow). This is an updated version of Figure 5 in Olausen et al. (2013) using the magnetar data from this work; data for other sources remains unchanged. As such, our observations and conclusions remain largely unchanged from the aforementioned paper: there is a general trend for higher- $B$ sources - of course the magnetars, but also high- $B$ radio pulsars to display greater blackbody temperatures than low- $B$ pulsars of similar age, suggesting that the magnetic field plays a role in the observed thermal properties of pulsars. For a more detailed discussion, see Olausen et al. (2013). Finally, note that SGR 0418+5729, despite having $B<10^{13} \mathrm{G}$, is set apart from the other low- $B$ sources by its much greater $k T$.

\subsection{Multiwavelength Properties}

Figure 15 shows a $P-\dot{P}$ diagram with radio pulsars, XINSs, and the magnetars indicated, as well as their detection status in soft X-rays, hard X-rays, and the radio band. From the plot it is clear that sources detected persistently in hard X-rays tend to be those with the highest $B$ fields $\left(10^{14.5}-10^{15} \mathrm{G}\right.$ and above) unless they are particularly distant, e.g. in the Magellanic Clouds. $4 \mathrm{U}$ 


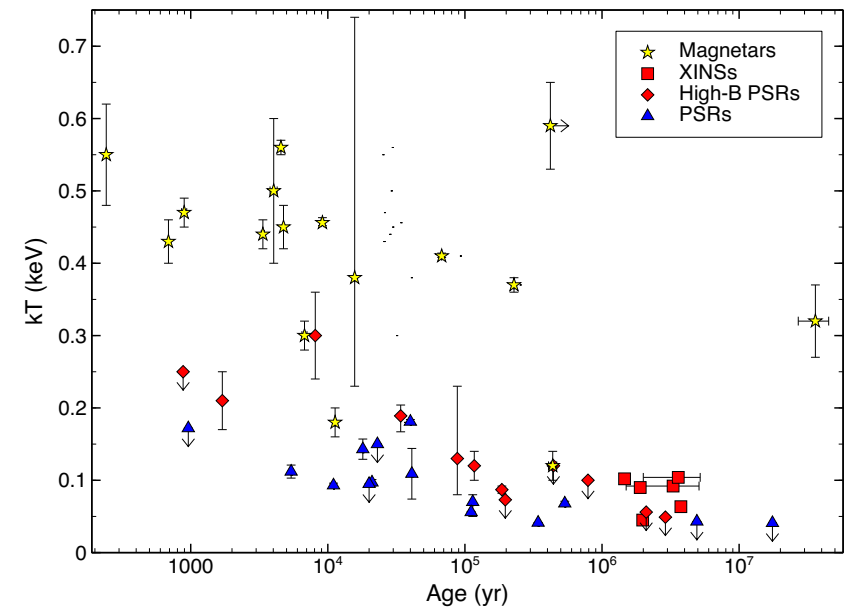

Figure 14. Blackbody temperatures vs. characteristic ages for magnetars (yellow stars), XINSs (red squares), high- $B$ pulsars ( $B \geq 10^{13} \mathrm{G}$; red diamonds), and normal pulsars (blue triangles). Data for the magnetars are taken from this work; for data on the other sources, see Olausen et al. (2013) and references therein, particularly Table 4 in Zhu et al. (2011).

$0142+61$ and $1 \mathrm{E} 2259+586$ are detected in hard X-rays but have somewhat lower $B$ fields; this further emphasizes their apparently outlier nature (noted above). Alternatively, from Figure 16, where the multiwavelength detections of the catalogued magnetars are shown as a function of quiescent $2-10 \mathrm{keV}$ X-ray flux $F_{\mathrm{X}}$, it is clear that only the sources with the highest $F_{\mathrm{X}}$ are detected persistently in hard X-rays. Moreover, hard X-rays are generally detected in sources in outburst, i.e. when the soft-X-ray flux is anomalously high. These facts suggest that all magnetars produce hard X-rays but that current hard X-ray missions do not have the sensitivity to detect them. NASA's NuSTAR mission (Harrison et al. 2013), the first focusing hard X-ray telescope, may help in this regard.

The radio emission observed from magnetars is strikingly different from the hard X-ray behavior. As is clear from Figure [16. radio emission has only been seen in sources with low $F_{\mathrm{X}}$ when in outburst in spite of extensive radio observations and stringent upper limits (see Table 5) of most of the objects catalogued including those with the largest values of $F_{\mathrm{X}}$ (Burgav et al. 2006b; Crawford et al. 2007; Lazarus et al. 2012; Tong et al. 2013; Archibald et al. 2013). ${ }^{5} \quad$ Although beaming may play a role (see discussion in Lazarus et al. 2012), with increasing statistics, the segregation of radio detections in the $P-\dot{P}$ diagram (Figure 15) is interesting.

Rea et al. (2012a) have suggested that there exists a 'fundamental plane' in magnetar spin and radiative phase space which distinguishes sources of different radio emission properties. Specifically, they argue on physical grounds that magnetars with high $\dot{E}$ and low $L_{\mathrm{X}}$ should be radio bright, while low $\dot{E}$, high $L_{\mathrm{X}}$ should not be radio detected. This is in principle an explanation for the striking asymmetry in the $P-\dot{P}$ distribution we see for radio-emitting sources. On the other hand, the recent non-detection of magnetar Swift J1834.9-0846, which

\footnotetext{
${ }^{5}$ We note claimed radio detections of $4 \mathrm{U} 0142+61,1 \mathrm{E} 2259+586$ and XTE J1810-197 (Malofeev et al. 2012) however the detections have not yet been confirmed using another observatory.
}

has $\dot{E} / L_{\mathrm{X}}$ where Rea et al. (2012a) would predict radio emission, argues against this picture (Tong et al. 2013; Esposito et al. 2013). Moreover, the 'fundamental plane' picture also predicts radio emission from the high- $B$ rotation-powered pulsar PSR J1846-0258, which in fact has been shown to be radio quiet (Archibald et al. 2008). Rea et al. (2012a) argue that a previously reported very large distance $(21 \mathrm{kpc})$ to the source (Becker \& Helfand 1984) together with its presence in supernova remnant Kes 75 could somehow hinder a radio detection, perhaps because of a high dispersion measure (DM). However, upto-date distance estimates for this pulsar (Leahy \& Tian 2008a; Su et al. 2009) place it significantly closer (5.1$10.9 \mathrm{kpc}$ ), and the system's hydrogen column density as measured with X-ray observations, $N_{\mathrm{H}}=2-4 \times 10^{22} \mathrm{~cm}^{-2}$ predicts, on the basis of an empirical DM vs $N_{\mathrm{H}}$ law (He et al. 2013), $D M \simeq 600-1300 \mathrm{pc} \mathrm{cm}^{-3}$. This is well within the range of observed DMs for radio pulsars, particularly those with only moderately fast rotation periods like the 0.326-s period of PSR J1846-0258. Hence we disagree with the conclusion of Rea et al. (2012a) that a radio detection of PSR J1846-0258 is difficult due to its environment. On the other hand, unfortunate radio beaming, as well as the episodic nature of radio emission from magnetars, may play a role for this pulsar and Swift J1834.9-0846. Continued radio observations of magnetars in outburst to increase statistics for radio emission in the population will be helpful for deciding whether $\dot{E} / L_{\mathrm{X}}$ plays a meaningful role in radio detectability of magnetars.

\section{CONCLUSIONS}

We have compiled the first catalog of all currently known magnetars, including 21 confirmed sources and 5 candidates. Where available from the literature we have provided spatial properties (coordinates, proper motion, distance, and proposed associations), timing data (period, period derivative, and derived parameters), spectral parameters for the quiescent soft X-ray emission, and observed properties or upper limits in the radio, infrared, optical, hard X-ray and gamma-ray bands. We note that the known magnetar population is relatively free from selection for location in the Galaxy thanks to the all-sky X-ray monitors that have found so many of these objects in recent years. We constructed histograms in Galactic longitude and latitude, spin period $P$, spin-inferred magnetic field $B$, spin-down luminosity $\dot{E}$, and characteristic age $\tau_{c}$, to compare the magnetar distributions with the distributions of the known pulsar population. We measure the scale height of magnetars for the first time and find it to be smaller than that of OB stars, supporting the hypothesis that the most massive $\mathrm{O}$ stars are magnetar progenitors. We note the relatively narrow ranges in $P$ and $B$ observed for the magnetars, which stand in contrast to the far wider ranges in $\dot{E}$ and $\tau_{c}$. That the characteristic age range is so broad in spite of so small a scale height for these objects argues that the former is generally a poor age indicator. We confirm correlations between $\Gamma$ and $B$, previously identified by Kaspi \& Boydstun (2010) and Enoto et al. (2010a), and $L_{\mathrm{X}}$ and $B$, previously noted by An et al. (2012), and observe an excluded region in the plot of $L_{\mathrm{X}}$ versus $\Gamma$. Finally, we find that detections of magnetars in the hard 


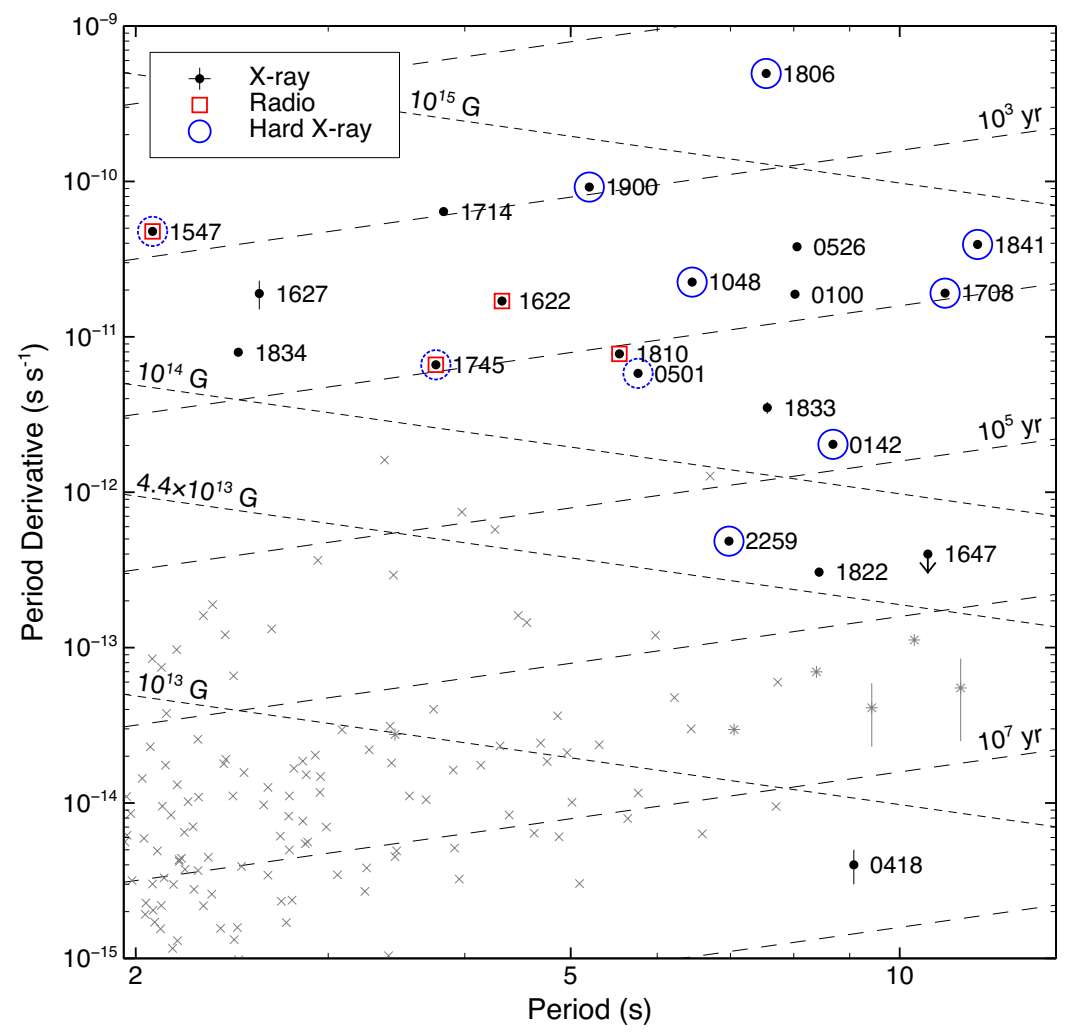

Figure 15. $P-\dot{P}$ diagram showing radio pulsars (crosses), XINSs (asterisks), and magnetars (circles). Radio-detected magnetars are marked with red squares. Blue circles denote magnetars that have been detected in the hard X-ray band $(>10 \mathrm{keV})$, with a dotted circle indicating that it has been so detected only in outburst.

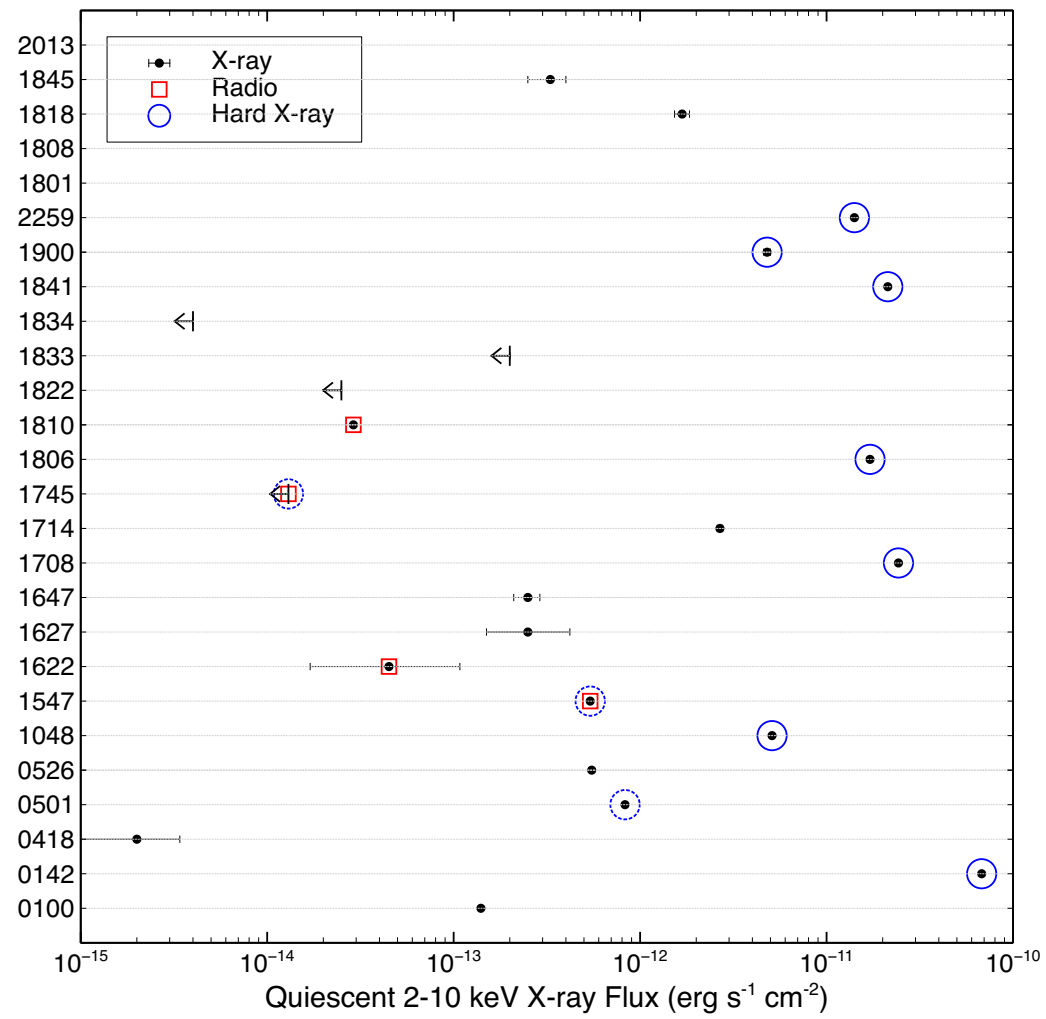

Figure 16. Magnetar detections as a function of quiescent $2-10 \mathrm{keV} \mathrm{X}$-ray flux $F_{\mathrm{X}}$. Radio and hard X-ray detections are marked by red squares and blue circles, as described for Figure 15 
X-ray seem to be correlated with soft X-ray flux and $B$, while radio detections show, if anything, the opposite trend. A regularly maintained online version of the catalog has been made available, with one main table focused on the timing and X-ray data, and two additional tables for alternative values and detailed records of optical and near-infrared observations. We plan to maintain this with regular updates as new magnetar results appear and encourage the community to provide feedback and suggestions for improvement on this constantly evolving initiative.

We thank J. Lazio for assistance in producing Figure2, as well as N. Murray, L. Drissen, J. Maíz Apellániz, C. Thompson, B. Gaensler, J. Halpern, S. Tendulkar, R. Duncan, and the McGill Pulsar Group for helpful discussions. We also thank K. Boydstun and C. Tam for their work on early versions of the online magnetar catalog, as well as the magnetar community for their past and continuing input. V.M.K. holds the Lorne Trottier Chair in Astrophysics and Cosmology, and a Canada Research Chair, and acknowledges additional support from an NSERC Discovery Grant and Accelerator Supplement, from FQRNT via le Centre de Recherche Astrophysique du Québec and the Canadian Institute for Advanced Research.

\section{APPENDIX}

\section{MAGNETAR NAMES}

In Table 8 we list the commonly used names (as well as a few less-common alternatives) of all catalogued magnetars along with our proposed new names. Said names follow the convention MG JHHMM+/-DDMM (here, 'MG' stands for 'magnetar') similar to the naming convention for pulsars, and indeed, for the sake of comparison, we also list the PSR names used by the ATNF catalog in Table 8. Note that this naming scheme is only used for confirmed magnetars, although for completeness we also list the five magnetar candidates in the Table.

\section{REFERENCES}

Abdo, A. A., Ackermann, M., Ajello, M., et al. 2010, ApJ, 725, L73

An, H., Kaspi, V. M., Archibald, R., \& Cumming, A. 2013a, ApJ, 763,82

An, H., Kaspi, V. M., Tomsick, J. A., et al. 2012, ApJ, 757, 68

An, H., Hascoet, R., Kaspi, V. M., et al. 2013b, ApJ, 779, 163

Anderson, G. E., Gaensler, B. M., Slane, P. O., et al. 2012, ApJ, 751,53

Archibald, A. M., Kaspi, V. M., Livingstone, M. A., \& McLaughlin, M. A. 2008, ApJ, 688, 550

Archibald, R. F., Kaspi, V. M., Ng, C.-Y., et al. 2013, Nature, 497,591

Bahcall, J. N. 1984, ApJ, 276, 156

Balman, S., Safi-Harb, S., Ibrahim, A. I., Swank, J. H., \& Markwardt, C. B. 2003, ATel, 195

Barthelmy, S. D., Barbier, L. M., Cummings, J. R., et al. 2005, Space Sci. Rev., 120, 143

Becker, R. H., \& Helfand, D. J. 1984, ApJ, 283, 154

Beloborodov, A. M. 2009, ApJ, 703, 1044

Bernardini, F., Israel, G., Stella, L., et al. 2011, A\&A, 529, 19

Bibby, J. L., Crowther, P. A., Furness, J. P., \& Clark, J. S. 2008, MNRAS, 386, L23

Bloom, J. S. 2005, GCN Circ., 4042

Burgay, M., Rea, N., Israel, G., \& Possenti, A. 2006a, ATel, 903

Burgay, M., Rea, N., Israel, G., et al. 2006b, MNRAS, 372, 410

Cameron, P. B., Chandra, P., Ray, A., et al. 2005, Nature, 434, 1112
Camilo, F., Ransom, S. M., Halpern, J. P., \& Reynolds, J. E. 2007a, ApJ, 666, L93

Camilo, F., Ransom, S. M., Halpern, J. P., et al. 2006, Nature, 442, 892

Camilo, F., Reynolds, J. E., Johnston, S., Halpern, J. P., \&

Ransom, S. M. 2008, ApJ, 679, 681

Camilo, F., Cognard, I., Ransom, S. M., et al. 2007b, ApJ, 663, 497

Camilo, F., Ransom, S. M., Peñalver, J., et al. 2007c, ApJ, 669, 561

Cline, T. L., Frederiks, D. D., Golenetskii, S., et al. 2000, ApJ, 531,407

Cline, T. L., Desai, U. D., Teegarden, B. J., et al. 1982, ApJ, 255, L45

Colpi, M., Geppert, U., \& Page, D. 2000, ApJ, 529, L29

Conti, P. S., \& Vacca, W. D. 1990, AJ, 100, 431

Corbel, S., Chapuis, C., Dame, T. M., \& Durouchoux, P. 1999, ApJ, 526, L29

Corbel, S., \& Eikenberry, S. S. 2004, A\&A, 419, 191

Cordes, J. M., \& Lazio, T. J. W. 2002, arXiv, arXiv:astro-ph/0207156

Crawford, F., Hessels, J. W. T., \& Kaspi, V. M. 2007, ApJ, 662, 1183

Davies, B., Figer, D. F., Kudritzki, R.-P., et al. 2009, ApJ, 707, 844

de Ugarte Postigo, A., Castro-Tirado, A. J., Covino, S., et al. 2009, A\&A, 500, 1157

Deller, A. T., Camilo, F., Reynolds, J. E., \& Halpern, J. P. 2012, ApJ, 748, L1

den Hartog, P. R., Kuiper, L., \& Hermsen, W. 2008a, A\&A, 489, 263

den Hartog, P. R., Kuiper, L., Hermsen, W., et al. 2008b, A\&A, 489,245

- 2007, Ap\&SS, 308, 647

Dhillon, V. S., Marsh, T. R., Hulleman, F., et al. 2005, MNRAS, 363,609

Dhillon, V. S., Marsh, T. R., Littlefair, S. P., et al. 2009, MNRAS, 394, L112

2011, MNRAS, 416, L16

Dib, R., Kaspi, V.' M., \& Gavriil, F. P. 2007, ApJ, 666, 1152

-. 2008, ApJ, 673, 1044

-. 2009, ApJ, 702, 614

Dib, R., Kaspi, V. M., Scholz, P., \& Gavriil, F. P. 2012, ApJ, 748, 3

Duncan, R. C., \& Thompson, C. 1992, ApJ, 392, L9

Duncan, R. C., \& Thompson, C. 1996, in AIP Conference Proceedings, Vol. 366, High Velocity Neutron Stars and Gamma-ray Bursts, ed. R. E. Rothschild \& R. E. Lingenfelter (Melville, NY: AIP), 111-117

Durant, M. 2005, ApJ, 632, 563

Durant, M., Kargaltsev, O. \& Pavlov, G. G. 2011, ApJ, 742, 77

Durant, M., \& van Kerkwijk, M. H. 2005a, ApJ, 628, L135

-. 2005b, ApJ, 627, 376

-. 2006a, ApJ, 650, 1070

-. 2006b, ApJ, 652, 576

—. 2006c, ApJ, 648, 534

-. 2008, ApJ, 680, 1394

Eatough, R. P., Falcke, H., Karuppusamy, R., et al. 2013, Nature, 501,391

Elias, F., Cabrera-Caño, J., \& Alfaro, E. J. 2006, AJ, 131, 2700

Enoto, T., Makishima, K., Nakazawa, K., et al. 2011, PASJ, 63, 387

Enoto, T., Nakazawa, K., Makishima, K., et al. 2010a, ApJ, 722, L162

-. 2010b, PASJ, 62, 475

Enoto, T., Rea, N., Nakagawa, Y. E., et al. 2010c, ApJ, 715, 665

Ertan, Ü., Alpar, M. A., Erkut, M. H., Ekși, K. Y., \& Calışkan, S. 2007, Ap\&SS, 308, 73

Ertan, Ü., Ekşi, K. Y., Erkut, M. H., \& Alpar, M. A. 2009, ApJ, 702,1309

Esposito, P., Mereghetti, S., Tiengo, A., et al. 2007, A\&A, 476 321

Esposito, P., Israel, G., Zane, S., et al. 2008, MNRAS, 390, L34

Esposito, P., Burgay, M., Possenti, A., et al. 2009a, MNRAS, 399, L44

Esposito, P., Tiengo, A., Mereghetti, S., et al. 2009b, ApJ, 690, L105

Esposito, P., Israel, G., Turolla, R., et al. 2010, MNRAS, 405, 1787

-. 2011, MNRAS, 416, 205

Esposito, P., Tiengo, A., Rea, N., et al. 2013, MNRAS, 429, 3123

Fahlman, G. G., \& Gregory, P. C. 1981, Nature, 293, 202

Fatkhullin, T., de Ugarte Postigo, A., Castro-Tirado, A. J., et al. 2008, GCN Circ., 8160

Faucher-Giguère, C.-A., \& Kaspi, V. M. 2006, ApJ, 643, 332

Figer, D. F., Najarro, F., Geballe, T. R., Blum, R. D., \& Kudritzki, R. P. 2005, ApJ, 622, L49 
Frail, D. A., Kulkarni, S. R., \& Bloom, J. S. 1999, Nature, 398, 127

Fuchs, Y., Mirabel, F., Chaty, S., et al. 1999, A\&A, 350, 891

Gaensler, B. M., \& Chatterjee, S. 2008, GCN Circ., 8149

Gaensler, B. M., Gotthelf, E. V., \& Vasisht, G. 1999, ApJ, 526, L37

Gaensler, B. M., McClure-Griffiths, N. M., Oey, M. S., et al. 2005, ApJ, 620, L95

Gavriil, F. P., Gonzalez, M. E., Gotthelf, E. V., et al. 2008, Science, 319, 1802

Gavriil, F. P., \& Kaspi, V. M. 2002, ApJ, 567, 1067

-. 2004, ApJ, 609, L67

Gavriil, F. P., Kaspi, V. M., \& Woods, P. M. 2002, Nature, 419 142

-. 2004, ApJ, 607, 959

Gelfand, J. D., \& Gaensler, B. M. 2007, ApJ, 667, 1111

Gies, D. R. 1987, ApJS, 64, 545

Göğüs, E., Woods, P. M., Kouveliotou, C., et al. 2010a, ApJ, 722 899

Göğüs, E., Cusumano, G., Levan, A. J., et al. 2010b, ApJ, 718, 331

Gotthelf, E. V., Halpern, J. P., Buxton, M., \& Bailyn, C. 2004, ApJ, 605, 368

Götz, D., Mereghetti, S., Tiengo, A., \& Esposito, P. 2006, A\&A, 449, L31

Götz, D., Rea, N., Israel, G., et al. 2007, A\&A, 475, 317

Guidorzi, C., Mundell, C. G., Gomboc, A., et al. 2005, GCN Circ., 4035

Haberl, F. 2007, Ap\&SS, 308, 181

Halpern, J. P. 2008, GCN Circ., 8129

Halpern, J. P., \& Gotthelf, E. V. 2010a, ApJ, 725, 1384

-. 2010b, ApJ, 710, 941

Harrison, F. A., Craig, W. W., Christensen, F. E., et al. 2013, ApJ, 770, 103

Haschke, R., Grebel, E. K., \& Duffau, S. 2012a, AJ, 144, 106

- 2012b, AJ, 144, 107

He, C., Ng, C.-Y., \& Kaspi, V. M. 2013, ApJ, 768, 64

Helfand, D. J., Chatterjee, S., Brisken, W. F., et al. 2007, ApJ, 662,1198

Hellier, C. 1994, MNRAS, 271, L21

Hessels, J. W. T., Rea, N., Ransom, S. M., \& Stappers, B. W. 2008, GCN Circ., 8134

Holland, S. T., \& Krimm, H. A. 2008, GCN Circ., 8325

Hulleman, F., Tennant, A. F., van Kerkwijk, M. H., et al. 2001, ApJ, 563, Lे49

Hulleman, F., van Kerkwijk, M. H., \& Kulkarni, S. R. 2004, A\&A, 416, 1037

Israel, G., Covino, S., Stella, L., et al. 2002, ApJ, 580, L143

Israel, G., Covino, S., Perna, R., et al. 2003, ApJ, 589, L93

Israel, G., Rea, N., Mangano, V., et al. 2004a, ApJ, 603, L97

Israel, G., Stella, L., Covino, S., et al. 2004b, in IAU Symposium, Vol. 218, Young Neutron Stars and Their Environments, ed. F. Camilo \& B. M. Gaensler (San Francisco: ASP), 247

Israel, G., Covino, S., Mignani, R. P., et al. 2005, A\&A, 438, L1

Israel, G., Rea, N., Rol, E., et al. 2009, ATel, 1909

Israel, G., Esposito, P., Rea, N., et al. 2010, MNRAS, 408, 1387

Joshi, Y. C. 2007, MNRAS, 378, 768

Kaneko, Y., Göğüs, E., Kouveliotou, C., et al. 2010, ApJ, 710 1335

Kaplan, D. L. 2008, in AIP Conference Proceedings, Vol. 983, 40 Years of Pulsars: Millisecond Pulsars, Magnetars, and More, ed. C. G. Bassa, Z. Wang, A. Cumming, \& V. M. Kaspi (Melville, NY: AIP), 331-339

Kaplan, D. L., Chakrabarty, D., Wang, Z., \& Wachter, S. 2009a, ApJ, 700, 149

Kaplan, D. L., Chatterjee, S., Hales, C. A., Gaensler, B. M., \& Slane, P. O. 2009b, AJ, 137, 354

Kaplan, D. L., Kulkarni, S. R., van Kerkwijk, M. H., et al. 2001, ApJ, 556, 399

Kargaltsev, O., Kouveliotou, C., Pavlov, G. G., et al. 2012, ApJ, 748,26

Kaspi, V. M. 2010, Publ. Natl. Acad. Sci. USA, 107, 7147

Kaspi, V. M., \& Boydstun, K. 2010, ApJ, 710, L115

Kaspi, V. M., Gavriil, F. P., Woods, P. M., et al. 2003, ApJ, 588, L93

Kaspi, V. M., \& McLaughlin, M. A. 2005, ApJ, 618, L41

Kaspi, V. M., Roberts, M. S. E., \& Harding, A. K. 2006, in Compact Stellar X-ray Sources, ed. W. H. G. Lewin \& M. van der Klis (Cambridge: Cambridge University Press), 279-339

Keith, M. J., Johnston, S., Levin, L., \& Bailes, M. 2011, MNRAS, 416,346

Klose, S., Stecklum, B., \& Laux, U. 2001, GCN Circ., 1044

Klose, S., Henden, A. A., Geppert, U., et al. 2004, ApJ, 609, L13

Kosugi, G., Ogasawara, R., \& Terada, H. 2005, ApJ, 623, L125

Kothes, R., \& Dougherty, S. M. 2007, A\&A, 468, 993

Kothes, R., \& Foster, T. 2012, ApJ, 746, L4
Kouveliotou, C., Dieters, S., Strohmayer, T., et al. 1998, Nature, 393,235

Kuiper, L., Hermsen, W., den Hartog, P. R., \& Collmar, W. 2006, ApJ, 645, 556

Kuiper, L., Hermsen, W., den Hartog, P. R., \& Urama, J. O.

2012, ApJ, 748, 133

Kulkarni, S. R., Kaplan, D. L., Marshall, H. L., et al. 2003, ApJ, 585,948

Kumar, H. S., \& Safi-Harb, S. 2010, ApJ, 725, L191

Lamb, D., Graziani, C., Shirasaki, Y., et al. 2003, GCN Circ., 2351

Lamb, R. C., Fox, D. W., Macomb, D. J., \& Prince, T. A. 2002, ApJ, 574, L29

Laros, J. G., Fenimore, E. E., Klebesadel, R. W., et al. 1987, ApJ, 320, L111

Lazarus, P. 2013, in IAU Symposium, Vol. 291, Neutron Stars and Pulsars: Challenges and Opportunities after 80 Years, ed. J. van Leeuwen (Cambridge University Press), 35-40

Lazarus, P., Kaspi, V. M., Champion, D. J., Hessels, J. W. T., \& Dib, R. 2012, ApJ, 744, 97

Leahy, D. A., \& Tian, W. W. 2007, A\&A, 461, 1013

-. 2008a, A\&A, 480, L25

-. 2008b, AJ, 135, 167

Levin, L., Bailes, M., Bates, S. D., et al. 2010, ApJ, 721, L33

Leyder, J. C., Walter, R., \& Rauw, G. 2008, A\&A, 477, L29

Lin, L., Kouveliotou, C., Baring, M. G., et al. 2011, ApJ, 739, 87

Maíz-Apellániz, J. 2001, AJ, 121, 2737

Maíz Apellániz, J., Alfaro, E. J., \& Sota, A. 2008, arXiv, arXiv:0804.2553

Malheiro, M., Rueda, J. A., \& Ruffini, R. 2012, PASJ, 64, 56

Malofeev, V. M., Teplykh, D. A., \& Logvinenko, S. V. 2012, Astron. Rep., 56, 35

Manchester, R. N., Hobbs, G. B., Teoh, A., \& Hobbs, M. 2005 , AJ, 129, 1993

Manchester, R. N., Lyne, A. G., Camilo, F., et al. 2001, MNRAS, 328,17

Marshall, F. E., \& Gelbord, J. M. 2010, GCN Circ., 10540

Mazets, E. P., \& Golenetskii, S. V. 1981, Ap\&SS, 75, 47

Mazets, E. P., Golenetskij, S. V., \& Guryan, Y. A. 1979a, Soviet Ast., 5, 343

Mazets, E. P., Golentskii, S. V., Il'Inskii, V. N., Aptekar, R. L., \& Guryan, I. A. 1979b, Nature, 282, 587

McGarry, M. B., Gaensler, B. M., Ransom, S. M., Kaspi, V. M., \& Veljkovik, S. 2005, ApJ, 627, L137

Meegan, C., Lichti, G., Bhat, N. P., et al. 2009, ApJ, 702, 791

Mereghetti, S. 2008, A\&A Rev., 15, 225

—. 2013, Braz. J. Phys.

Mereghetti, S., Esposito, P., Tiengo, A., et al. 2012, A\&A, 546, 30

Mereghetti, S., Götz, D., Mirabel, I. F., \& Hurley, K. 2005, A\&A, 433, L9

Mereghetti, S., \& Stella, L. 1995, ApJ, 442, L17

Mereghetti, S., Esposito, P., Tiengo, A., et al. 2006, ApJ, 653, 1423

Mignani, R. P., Rea, N., Testa, V., et al. 2009, A\&A, 497, 451

Milhalas, D., \& Binney, J. 1981, Galactic Astronomy: Structure and Kinematics, 2nd edn. (San Francisco: Freeman)

Minter, A. H., Camilo, F., Ransom, S. M., Halpern, J. P., \& Zimmerman, N. 2008, ApJ, 676, 1189

Molkov, S. V., Hurley, K., Sunyaev, R. A., et al. 2005, A\&A, 433, L13

Mori, K., Gotthelf, E. V., Zhang, S., et al. 2013, ApJ, 770, L23

Morii, M., Kawai, N., Kataoka, J., et al. 2005, Adv. Space Res., 35,1177

Morii, M., Kitamoto, S., Shibazaki, N., et al. 2010, PASJ, 62, 1249

Muno, M. P., Clark, J. S., Crowther, P. A., et al. 2006, ApJ, 636, L41

Nakagawa, Y. E., Mihara, T., Yoshida, A., et al. 2009, PASJ, 61, 387

Nakamura, R., Bamba, A. Ishida, M., et al. 2009, PASJ, 61, 197

Olausen, S. A., Kaspi, V. M., Lyne, A. G., \& Kramer, M. 2010, ApJ, 725, 985

Olausen, S. A., Zhu, W., Vogel, J. K., et al. 2013, ApJ, 764, 1

Ouyed, R., Leahy, D., \& Niebergal, B. 2007a, A\&A, 473, 357

-. 2007b, A\&A, 475, 63

Pagani, C., Beardmore, A. P., \& Kennea, J. A. 2011, ATel, 3493

Palaniswamy, D., Bhat, N. D. R., Tingay, S. J., et al. 2013, ATel, 5076

Park, S., Hughes, J. P., Slane, P. O., et al. 2012, ApJ, 748, 117

Pons, J. A., Miralles, J. A., \& Geppert, U. 2009, A\&A, 496, 207

Popov, S. B., Pons, J. A., Miralles, J. A., Boldin, P. A., \&

Posselt, B. 2010, MNRAS, 401, 2675

Qiu, Y., Lu, C. L., Lou, Y. Q., Urata, Y., \& Huang, K. Y. 2005, GCN Circ., 4036

Rea, N., \& Esposito, P. 2011, in High-Energy Emission from Pulsars and their Systems, ed. D. F. Torres \& N. Rea, Astrophysics and Space Science Proceedings (Berlin: Springer), 247 
Rea, N., Pons, J. A., Torres, D. F., \& Turolla, R. 2012a, ApJ, 748, L12

Rea, N., Testa, V., Israel, G., et al. 2004, A\&A, 425, L5

Rea, N., Nichelli, E., Israel, G., et al. 2007a, MNRAS, 381, 293

Rea, N., Israel, G., Oosterbroek, T., et al. 2007b, Ap\&SS, 308, 505

Rea, N., Israel, G., Turolla, R., et al. 2009, MNRAS, 396, 2419

Rea, N., Esposito, P., Turolla, R., et al. 2010, Science, 330, 944

Rea, N., Israel, G., Esposito, P., et al. 2012b, ApJ, 754, 27

Rea, N., Esposito, P., Pons, J. A., et al. 2013a, ApJ, 775, L34

Rea, N., Israel, G., Pons, J. A., et al. 2013b, ApJ, 770, 65

Reed, B. C. 1997, PASP, 109, 1145

-. 2000, AJ, 120, 314

Rickett, B. J. 1990, ARA\&A, 28, 561

Rosen, S., Holland, S. T., Marshall, F. E., Boyd, P., \& Gehrels, N. 2005, GCN Circ., 4038

Sakamoto, T., Barbier, L., Barthelmy, S. D., et al. 2011, Adv. Space Res., 47, 1346

Sasaki, M., Plucinsky, P. P., Gaetz, T. J., \& Bocchino, F. 2013, A\&A, 552, 45

Sato, T., Bamba, A., Nakamura, R., \& Ishida, M. 2010, PASJ, 62, L33

Scholz, P., \& Kaspi, V. M. 2011, ApJ, 739, 94

Scholz, P., Ng, C.-Y., Livingstone, M. A., et al. 2012, ApJ, 761, 66

Shannon, R. M., \& Johnston, S. 2013, MNRAS, 435, L29

Spitler, L. G., Lee, K. J., Eatough, R. P., et al. 2014, ApJ, 780, L3

Su, Y., Chen, Y., Yang, J., et al. 2009, ÄpJ, 694, 376

Tam, C. R., Gavriil, F. P., Dib, R., et al. 2008, ApJ, 677, 503

Tam, C. R., Kaspi, V. M., Gaensler, B. M., \& Gotthelf, E. V. 2006, ApJ, 652, 548

Tam, C. R., Kaspi, V. M., van Kerkwijk, M. H., \& Durant, M. 2004, ApJ, 617, L53

Tanvir, N. R., \& Varricatt, W. 2008, GCN Circ., 8126

Tello, J. C., Sota, A., \& Castro-Tirado, A. J. 2011, GCN Circ., 12272

Tendulkar, S. P. 2013, in IAU Symposium, Vol. 291, Proceedings of the International Astronomical Union, ed. J. van Leeuwen (Cambridge University Press), 514-516

Tendulkar, S. P., Cameron, P. B., \& Kulkarni, S. R. 2012, ApJ, 761,76

-. 2013, ApJ, 772, 31

Testa, V., Rea, N., Mignani, R. P., et al. 2008, A\&A, 482, 607

Thompson, C., \& Duncan, R. C. 1995, MNRAS, 275, 255

-. 1996, ApJ, 473, 322

Thompson, C., Lyutikov, M., \& Kulkarni, S. R. 2002, ApJ, 574, 332

Tian, W. W., \& Leahy, D. A. 2008, ApJ, 677, 292

-. 2012, MNRAS, 421, 2593
Tian, W. W., Li, Z., Leahy, D. A., \& Wang, Q. D. 2007, ApJ, 657, L25

Tiengo, A., Esposito, P., \& Mereghetti, S. 2008, ApJ, 680, L133

Tiengo, A., Esposito, P., Mereghetti, S., et al. 2009, MNRAS, 399, L74

Tiengo, A., Vianello, G., Esposito, P., et al. 2010, ApJ, 710, 227

Tong, H., Yuan, J.-P., \& Liu, Z.-Y. 2013, Res. Astron. Astrophys., 13, 835

Torii, K., Kinugasa, K., Katayama, K., Tsunemi, H., \& Yamauchi, S. 1998, ApJ, 503, 843

van der Horst, A. J., Connaughton, V., Kouveliotou, C., et al. 2010, ApJ, 711, L1

van Paradijs, J., Taam, R. E., \& van den Heuvel, E. P. J. 1995, A\&A, 299, L41

Vasisht, G., \& Gotthelf, E. V. 1997, ApJ, 486, L129

Vigano, D., Rea, N., Pons, J. A., et al. 2013, MNRAS, 434, 123

Vrba, F. J., Henden, A. A., Luginbuhl, C. B., et al. 2000, ApJ, $533, \mathrm{~L} 17$

Wachter, S., Ramirez-Ruiz, E., Dwarkadas, V. V., et al. 2008,

Nature, 453, 626

Wachter, S., Patel, S. K., Kouveliotou, C., et al. 2004, ApJ, 615, 887

Wang, Z., Bassa, C. G., Kaspi, V. M., Bryant, J. J., \& Morrell, N. 2008a, ApJ, 679, 1443

Wang, Z., \& Chakrabarty, D. 2002, ApJ, 579, L33

Wang, Z., Chakrabarty, D., \& Kaplan, D. L. 2008b, in AIP Conference Proceedings, Vol. 983, 40 Years of Pulsars: Millisecond Pulsars, Magnetars, and More, ed. C. G. Bassa, Z. Wang, A. Cumming, \& V. M. Kaspi (Melville, NY: AIP), $274-276$

Wang, Z., \& Kaspi, V. M. 2008, ApJ, 675, 695

Wang, Z., Kaspi, V. M., \& Higdon, S. J. U. 2007, ApJ, 665, 1292

Wang, Z., Kaspi, V. M., Osip, D., et al. 2006, ATel, 910

Woods, P. M., \& Thompson, C. 2006, in Compact Stellar X-ray Sources, ed. W. H. G. Lewin \& M. van der Klis (Cambridge: Cambridge University Press), 547-586

Woods, P. M., Kouveliotou, C., van Paradijs, J., et al. 1999, ApJ, 519, L139

Woods, P. M., Kouveliotou, C., Gavriil, F. P., et al. 2005, ApJ, 629,985

Younes, G., Kouveliotou, C., Kargaltsev, O., et al. 2012, ApJ, 757,39

Zhu, W., Kaspi, V. M., Dib, R., et al. 2008, ApJ, 686, 520

Zhu, W., Kaspi, V. M., McLaughlin, M. A., et al. 2011, ApJ, 734, 44 
Table 1

Magnetar Positions and Proper Motions

\begin{tabular}{|c|c|c|c|c|c|c|c|}
\hline Name & $\begin{array}{c}\text { Right Ascension }{ }^{\mathrm{a}} \\
(\mathrm{J} 2000)\end{array}$ & $\begin{array}{l}\text { Declination }^{\mathrm{a}} \\
\quad(\mathrm{J} 2000)\end{array}$ & $\begin{array}{c}l \\
\left(^{\circ}\right)\end{array}$ & $\begin{array}{c}b \\
\left(^{\circ}\right)\end{array}$ & $\begin{array}{c}\mu_{\mathrm{RA}}{ }^{\mathrm{b}} \\
\left(\operatorname{mas~yr}^{-1}\right)\end{array}$ & $\begin{array}{c}\mu_{\text {Dec }}{ }^{\mathrm{b}} \\
\left(\operatorname{mas~yr}^{-1}\right)\end{array}$ & References \\
\hline CXOU J010043.1-721134 & $010043.14(13)$ & $-721133.8(6)$ & 301.93 & -44.92 & $\ldots$ & $\ldots$ & 1 \\
\hline $4 \mathrm{U} 0142+61$ & $014622.407(28)^{\mathrm{c}}$ & $+614503.19(20)^{\mathrm{c}}$ & 129.38 & -0.43 & $-5.6(1.3)$ & $2.9(1.3)$ & 2,3 \\
\hline SGR $0418+5729$ & $041833.867(43)$ & $+573222.91(35)$ & 147.98 & +5.12 & $\ldots$ & $\ldots$ & 4 \\
\hline SGR $0501+4516$ & $050106.76(1)$ & $+451633.92(11)$ & 161.55 & +1.95 & $\ldots$ & $\ldots$ & 5 \\
\hline SGR $0526-66$ & $052600.89(10)$ & $-660436.3(6)$ & 276.09 & -33.25 & $\ldots$ & $\ldots$ & 6 \\
\hline 1E $1048.1-5937$ & $105007.14(8)$ & $-595321.4(6)$ & 288.26 & -0.52 & $\ldots$ & $\ldots$ & 7 \\
\hline 1E $1547.0-5408$ & $155054.12386(64)^{\mathrm{d}}$ & $-541824.1141(20)^{\mathrm{d}}$ & 327.24 & -0.13 & $4.8(5)^{\mathrm{f}}$ & $-7.9(3)^{\mathrm{f}}$ & 8 \\
\hline PSR J1622-4950 & $162244.89(8)$ & $-495052.7(8)$ & 333.85 & -0.10 & $\ldots$ & $\ldots$ & 9 \\
\hline SGR $1627-41$ & $163551.844(20)$ & $-473523.31(20)$ & 336.98 & -0.11 & $\cdots$ & $\cdots$ & 10 \\
\hline CXOU J164710.2-455216 & $164710.20(3)$ & -4552 16.90(30) & 339.55 & -0.43 & $\ldots$ & $\cdots$ & 11 \\
\hline 1RXS J170849.0-400910 & $170846.87(6)$ & $-400852.44(70)$ & 346.48 & +0.04 & $\cdots$ & $\cdots$ & 12 \\
\hline CXOU J171405.7-381031 & $171405.74(5)$ & $-381030.9(6)$ & 348.68 & +0.37 & $\ldots$ & $\cdots$ & 13 \\
\hline SGR J1745-2900 & $174540.164(2)^{\mathrm{d}}$ & $-290029.818(90)^{\mathrm{d}}$ & 359.94 & -0.05 & $\ldots$ & $\ldots$ & 14 \\
\hline SGR $1806-20$ & $180839.337(4)^{\mathrm{c}}$ & $-202439.85(6)^{\mathrm{c}}$ & 10.00 & -0.24 & $-4.5(1.4)$ & $-6.9(2.0)$ & 15,16 \\
\hline XTE J1810-197 & $180951.08696(28)^{\mathrm{d}}$ & $-194351.9315(40)^{\mathrm{d}}$ & 10.73 & -0.16 & $-6.60(6)^{f}$ & $-11.72(1.03)^{\mathrm{f}}$ & 17 \\
\hline Swift J1822.3-1606 & $182218.00(12)$ & $-160426.8(1.8)$ & 15.35 & -1.02 & $\ldots$ & $\ldots$ & 18 \\
\hline SGR $1833-0832$ & $183344.37(3)$ & $-083107.5(4)$ & 23.34 & +0.02 & $\ldots$ & $\cdots$ & 19 \\
\hline Swift J1834.9-0846 & $183452.118(40)$ & $-084556.02(60)$ & 23.25 & -0.34 & $\ldots$ & $\ldots$ & 20 \\
\hline $1 \mathrm{E} 1841-045$ & $1841 \quad 19.343(20)$ & $-045611.16(30)$ & 27.39 & -0.01 & $<4$ & $<4$ & 10,21 \\
\hline SGR $1900+14$ & $190714.33(1)^{\mathrm{d}^{\prime}}$ & +09 $1920.1(2)^{\mathrm{d}^{\prime}}$ & 43.02 & +0.77 & $-2.1(4)$ & $-0.6(5)$ & 22,16 \\
\hline 1E $2259+586$ & $230108.295(77)$ & $+585244.45(60)$ & 109.09 & -1.00 & $-9.9(1.1)$ & $-3.0(1.1)$ & 23,3 \\
\hline SGR $1801-23$ & $180059^{\mathrm{e}}$ & $-225648^{\mathrm{e}}$ & 6.91 & +0.07 & $\cdots$ & $\cdots$ & 24 \\
\hline SGR $1808-20$ & $180811.2(29.5)$ & $-203849(414)$ & 9.74 & -0.26 & $\ldots$ & $\ldots$ & 25 \\
\hline AX J1818.8-1559 & $181851.38(4)$ & $-155922.62(60)$ & 15.04 & -0.25 & $\ldots$ & $\ldots$ & 26 \\
\hline AX $1845.0-0258$ & $184454.68(4)$ & $-025653.1(6)$ & 29.56 & +0.11 & $\ldots$ & $\ldots$ & 27 \\
\hline SGR 2013+34 & $201356.9(7.3)$ & $+341948(90)$ & 72.32 & -0.10 & $\cdots$ & $\ldots$ & 28 \\
\hline
\end{tabular}

References. - (1) Lamb et al. (2002); (2) Hulleman et al. (2004); (3) Tendulkar et al. (2013); (4) van der Horst et al. (2010); (5)

Göğüs et al. (2010a); (6) Kulkarni et al. (2003); (7) Wang \& Chakrabartv (2002); (8) Deller et al. (2012); (9) Anderson et al. (2012);

(2013); (15) Israel et al. ; (2005); (16) Tendulkar et (12) Israel et al. (2003); (13) Halpern \& Gothell (2010a); (14) Shannon \& Johnston (2010b); (20) Kargaltsev et al.' (2012); (21) Tendulkar (2013); (22) Frail et al. (1999); (23) Hulleman et al. (2001); (24) Cline et al. (2000); (25) Lamb et al. (2003); (26) Mereghetti et al. (2012); (27) Tam et al. (2006); (28) Sakamoto et al. (2011)

Note. - In this and all subsequent tables, the unconfirmed, candidate magnetars are separated from the confirmed magnetars by a horizontal line.

${ }^{\text {a }}$ Positions are of the X-ray source unless otherwise specified.

b Proper motions have been corrected for Galactic rotation unless otherwise specified.

Position of the near-infrared counterpart.

d Position of the radio counterpart.

e See reference for the size and shape of the error box.

${ }^{\mathrm{f}}$ Proper motion in the sky frame. 
Table 2

Magnetar Timing Properties

\begin{tabular}{|c|c|c|c|c|c|c|c|c|c|}
\hline Name & $\begin{array}{l}P \\
(\mathrm{~s})\end{array}$ & $\begin{array}{l}\text { Epoch } \\
\text { (MJD) }\end{array}$ & $\begin{array}{c}\dot{P} \\
\left(10^{-11} \mathrm{~s} \mathrm{~s}^{-1}\right)\end{array}$ & $\begin{array}{l}\dot{P} \text { Range } \\
\text { (MJD) }\end{array}$ & Method $^{\mathrm{a}}$ & $\begin{array}{c}B \\
\left(10^{14} \mathrm{G}\right)\end{array}$ & $\begin{array}{c}\dot{E} \\
\left(10^{33} \mathrm{erg} \mathrm{s}^{-1}\right)\end{array}$ & $\begin{array}{c}\tau_{\mathrm{c}} \\
(\mathrm{kyr})\end{array}$ & References \\
\hline CXOU J010043.1-721134 & $8.020392(9)$ & 53032 & $1.88(8)$ & $52044-53033$ & A & 3.9 & 1.4 & 6.8 & 1 \\
\hline $4 \mathrm{U} 0142+61$ & $8.68832877(2)$ & 51704 & $0.20332(7)$ & $51610-53787$ & ED & 1.3 & 0.12 & 68 & 2 \\
\hline SGR $0418+5729$ & $9.07838822(5)$ & 54993 & $0.0004(1)$ & $54993-56164$ & $\mathrm{E}$ & 0.061 & 0.00021 & 36000 & 3 \\
\hline SGR $0501+4516$ & $5.76209653(3)$ & 54750 & $0.582(3)$ & $54700-54940$ & ED & 1.9 & 1.2 & 16 & 4 \\
\hline SGR 0526-66 & $8.0544(2)$ & 54414 & $3.8(1)$ & $52152-54414$ & $\mathrm{~A}$ & 5.6 & 2.9 & 3.4 & 5 \\
\hline 1E $1048.1-5937$ & $6.4578754(25)$ & 54185.9 & $\sim 2.25$ & $50473-54474$ & A & 3.9 & 3.3 & 4.5 & 6 \\
\hline 1E $1547.0-5408$ & $2.0721255(1)$ & 54854 & $\sim 4.77$ & $54743-55191$ & A & 3.2 & 210 & 0.69 & 7 \\
\hline PSR J1622-4950 & $4.3261(1)$ & 55080 & $1.7(1)$ & $54939-55214$ & A & 2.7 & 8.3 & 4.0 & 8 \\
\hline SGR $1627-41$ & $2.594578(6)$ & 54734 & $1.9(4)$ & $54620-54736$ & A & 2.2 & 43 & 2.2 & 9,10 \\
\hline CXOU J164710.2-455216 & $10.610644(17)$ & 53999.1 & $<0.04$ & $53513-55857$ & A & $<0.66$ & $<0.013$ & $>420$ & 11 \\
\hline 1RXS J170849.0-400910 & $11.003027(1)$ & 53635.7 & $1.91(4)$ & $53638-54015$ & ED & 4.6 & 0.57 & 9.1 & 12 \\
\hline CXOU J171405.7-381031 & $3.825352(4)$ & 55272 & $6.40(5)$ & $54856-55272$ & A & 5.0 & 45 & 0.95 & 13 \\
\hline SGR J1745-2900 & $3.7635537(2)$ & 56424.6 & $0.661(4)$ & $56406-56480$ & $\mathrm{E}$ & 1.6 & 4.9 & 9.0 & 14 \\
\hline SGR $1806-20$ & $7.547728(17)$ & 53097.5 & $\sim 49.5$ & $52021-53098$ & A & 20 & 45 & 0.24 & 15 \\
\hline XTE J1810-197 & $5.5403537(2)$ & 54000 & $0.777(3)$ & $53850-54127$ & $\mathrm{E}$ & 2.1 & 1.8 & 11 & 16 \\
\hline Swift J1822.3-1606 & $8.43771958(6)$ & 55761 & $0.0306(21)$ & $55758-55991$ & ED & 0.51 & 0.020 & 440 & 17 \\
\hline SGR $1833-0832$ & $7.5654084(4)$ & 55274 & $0.35(3)$ & $55274-55499$ & $\mathrm{ED}$ & 1.6 & 0.32 & 34 & 18 \\
\hline Swift J1834.9-0846 & $2.4823018(1)$ & 55783 & $0.796(12)$ & $55782-55812$ & $\mathrm{E}$ & 1.4 & 21 & 4.9 & 19 \\
\hline $1 \mathrm{E} 1841-045$ & $11.782898(1)$ & 53824 & $3.93(1)$ & $53828-53983$ & $\mathrm{E}$ & 6.9 & 0.95 & 4.7 & 12 \\
\hline SGR $1900+14$ & $5.19987(7)$ & 53826 & $9.2(4)$ & $53634-53826$ & $\mathrm{~A}$ & 7.0 & 26 & 0.90 & 20 \\
\hline $1 \mathrm{E} 2259+586$ & $6.978948446(4)$ & 51995.6 & $0.048430(8)$ & $50356-52016$ & ED & 0.59 & 0.056 & 230 & 21 \\
\hline SGR $1801-23$ & 年 & $\cdots$ & $\cdots$ & $\cdots$ & $\cdots$ & $\cdots$ & $\cdots$ & $\cdots$ & \\
\hline SGR $1808-20$ & $\cdots$ & $\cdots$ & $\cdots$ & $\cdots$ & $\cdots$ & $\cdots$ & $\cdots$ & $\cdots$ & $\cdots$ \\
\hline AX J1818.8-1559 & $\ldots$ & $\ldots$ & $\cdots$ & .. & $\ldots$ & $\ldots$ & $\ldots$ & .. & $\cdots$ \\
\hline AX $1845.0-0258$ & $6.97127(28)$ & 49272 & $\ldots$ & $\cdots$ & $\ldots$ & $\ldots$ & $\ldots$ & $\ldots$ & 22 \\
\hline SGR 2013+34 & & & $\cdots$ & $\cdots$ & $\cdots$ & $\ldots$ & $\cdots$ & $\cdots$ & \\
\hline
\end{tabular}

References. - (1) McGarry et al. (2005); (2) Dib et al. (2007); (3) Rea et al. (2013b); (4) Göŏüs et al. (2010a); (5) Tiengo et al. (2009); (6) Dib et al. (2009); (7) Dib et al. (2012); (8) Levin et al. (2010); (9) Esposito et al. (2009b); (10) Esposito et al. (2009a); (11) An et al. (2013a); (12) Dib et al. (2008); (13) Sato et al. (2010); (14) Rea et al. (2013a); (15) Nakagawa et al. (2009); (16) Camilo et al. (2007b); (17) Scholz et al. (2012); (18) Esposito et al. (2011); (19) Kargaltsev et al. (2012); (20) Mereghetti et al. (2006); (21) Gavriil \& Kaspi (2002); (22) Torii et al. (1998)

a Method by which $\dot{P}$ was measured. A: Long-term average, E: Phase-coherent timing ephemeris. ED: Phase-coherent timing ephemeris with additional higher derivatives.

b Other timing solutions with lower $\dot{P}$ are given in Rea et al. (2012b) and Scholz et al. (2012). 
Table 4

Optical and Near-Infrared Counterparts of Magnetars

\begin{tabular}{|c|c|c|c|c|c|c|c|c|c|}
\hline Name & $K_{s}$ & $H$ & $J$ & $I$ & $R$ & $V$ & $B$ & $U$ & References \\
\hline CXOU J010043.1-721134 & $\ldots$ & $\ldots$ & $\ldots$ & $>25.9$ & $\ldots$ & $24.2->26.2$ & $>25.6$ & $>24.2$ & 1,2 \\
\hline $4 \mathrm{U} 0142+61$ & $19.7-20.8$ & $20.5-20.9$ & $22.0-22.2$ & $23.4-24.0^{\mathrm{c}}$ & $24.9-25.6$ & $25.3-26.1$ & $27.2-28.1$ & $>25.8$ & $3-6$ \\
\hline SGR $0418+5729$ & $>19.6$ & $\ldots$ & $>27.4$ & $>25.1$ & $>24$ & $>28.6$ & $\ldots$ & $\ldots$ & $7-10$ \\
\hline SGR $0501+4516$ & $18.6-19.7^{\mathrm{c}}$ & $\ldots$ & $\ldots$ & $23.3-24.4^{\mathrm{c}}$ & $>23.0$ & $\ldots$ & $>26.9$ & $>24.7$ & $11-14$ \\
\hline SGR $0526-66$ & $\ldots$ & $\ldots$ & $\ldots$ & $>26.7$ & $\ldots$ & $>26.6$ & $>24.7$ & $>25.0$ & 15 \\
\hline $1 \mathrm{E} 1048.1-5937$ & $19.4-21.5$ & $20.8->23.3$ & $21.7->25.0$ & $24.9-26.2^{\mathrm{c}}$ & $>26.0$ & $>25.5$ & $>27.6$ & $>25.7$ & $16-21$ \\
\hline $1 \mathrm{E} 1547.0-5408^{\mathrm{a}}$ & $18.5^{->}>21.7$ & $\ldots$ & $\ldots$ & $\ldots$ & $\ldots$ & $>20.4$ & $>20.7$ & $>20.3$ & $22-24$ \\
\hline PSR J1622-4950 & $>20.7$ & 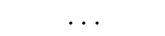 & $\ldots$ & ... & $\ldots$ & $\ldots$ & $\ldots$ & $\ldots$ & 25 \\
\hline SGR $1627-41$ & $\geq 19.1^{*}$ & $>19.5$ & $>21.5$ & ... & $\ldots$ & $\ldots$ & $\ldots$ & $\ldots$ & 26,27 \\
\hline CXOU J164710.2-455216 & $>21$ & & & $\ldots$ & $\ldots$ & $\cdots$ & $\cdots$ & $\cdots$ & 28 \\
\hline 1RXS J170849.0-400910b & $\geq 18.9^{*}$ & $\geq 20.0^{*}$ & $\geq 21.9^{*}$ & $>25.1$ & $>26.5$ & $\ldots$ & $\ldots$ & $\ldots$ & $29-31$ \\
\hline CXOU J171405.7-381031 & $\ldots$ & $\ldots$ & $\ldots$ & $\ldots$ & $\ldots$ & $\ldots$ & $\ldots$ & $\ldots$ & $\ldots$ \\
\hline SGR J1745-2900 & $\ldots$ & $\ldots$ & $\ldots$ & $\ldots$ & $\ldots$ & $\ldots$ & $\ldots$ & $\ldots$ & \\
\hline SGR $1806-20$ & $19.3-21.9$ & $>19.5$ & $>21.2$ & $\ldots$ & $>21.5$ & $\ldots$ & $\ldots$ & .. & $32-34$ \\
\hline XTE J1810-197 & $20.8-21.9$ & $21.5-22.7$ & $22.9-23.9$ & $>24.3$ & $>21.5$ & $>22.5$ & $\ldots$ & ... & $31,34-38$ \\
\hline Swift J1822.3-1606 & $>17.3$ & $>18.3$ & $>19.3$ & $>22.2$ & $\ldots$ & $\ldots$ & $\ldots$ & $\ldots$ & 39 \\
\hline SGR $1833-0832$ & $>22.4$ & $\ldots$ & $\ldots$ & $>24.9$ & $\ldots$ & $>21.4$ & $>21.3$ & $>22.3$ & 40,41 \\
\hline Swift J1834.9-0846 & $>19.5$ & $\ldots$ & $\ldots$ & $>21.6$ & $\ldots$ & $\ldots$ & $\ldots$ & $\ldots$ & 42,43 \\
\hline $1 \mathrm{E} 1841-045^{\mathrm{a}}$ & $19.6-20.5$ & $20.8->21.5$ & $>22.1$ & $\ldots$ & $\cdots$ & $\cdots$ & $\cdots$ & $\ldots$ & 31,44 \\
\hline SGR $1900+14^{\mathrm{a}}$ & $19.2-19.7$ & $\ldots$ & & $>21$ & $\ldots$ & $\ldots$ & $\ldots$ & $\ldots$ & 31,45 \\
\hline $1 \mathrm{E} 2259+586$ & $20.4-21.7$ & $\ldots$ & $>23.8$ & $>25.6$ & $>26.4$ & $\ldots$ & $\ldots$ & $\ldots$ & $46-48$ \\
\hline SGR $1801-23$ & $\cdots$ & $\cdots$ & $\cdots$ & $\cdots$ & $\cdots$ & $\cdots$ & $\cdots$ & $\cdots$ & $\cdots$ \\
\hline SGR 1808-20 & $\ldots$ & $\ldots$ & $\ldots$ & $\ldots$ & $\ldots$ & $\ldots$ & $\ldots$ & $\ldots$ & $\ldots$ \\
\hline AX J1818.8-1559 & $>17$ & $\ldots$ & $\ldots$ & $\ldots$ & $\ldots$ & $\ldots$ & $\ldots$ & $\ldots$ & 49 \\
\hline AX $1845.0-0258$ & $\ldots$ & $>21$ & $\ldots$ & $\ldots$ & $\ldots$ & $\ldots$ & $\ldots$ & $\ldots$ & 50 \\
\hline SGR 2013+34 & $>18.3$ & $>18.5$ & $>19.3$ & $>20.6$ & $>19$ & $>20.2$ & $>21.8$ & $>21.2$ & $51-54$ \\
\hline
\end{tabular}

References. - (1) Durant \& van Kerkwijk (2005a); (2) Durant \& van Kerkwijk (2008); (3) Hulleman et al. (2004); (4) Dhillon et al. (2005); (5) Morii et al. (2005); (6) Durant \& van Kerkwiik (2006b); (7) van der Horst et al. (2010); (8) Esposito et al. (2010); (9) Durant et al. (2011); (10) Rea et al. (2013b); (11) Tanvir \& Varricatt (2008); (12) Halpern (2008); (13) Fatkhullin et al. (2008); (14) Dhillon et al. (2011); (15) Kaplan et al. (2001); (16) Israel et al. (2002); (17) Wang \& Chakrabartv (2002); (18) Durant \& van Kerkwiik (2005b); (19) Tam et al. 2009); (25) Wang et al. (2008a); (26) Wachter et al. (2004); (27) Ho Ugarte Postigo et al. (2009): (28) Wang et al. (2006); (29) Israel et al. (2003); (30) Durant \& van Kerkwijk (2006c); (31) Testa et al. (2008); (32) Kosugi et al. (2005); (33) Israel et al. (2005); (34) Balman et al. (2003); (35) Gotthelf et al. (2004); (36) Israel et al. (2004a); (37) Rea et al. (2004); (38) Camilo et al. (2007c); (39) Rea et al. (2012b); (40) Marshall \& Gelbord (2010); (41) Gööüs et al. (2010b); (42) Tello et al. (2011); (43) Kargaltsev et al. (2012); (44) Durant (2005); (45) Klose et al. (2001); (46) Hulleman et al. (2001); (47) Kaspi et al. (2003) (48) Tam et al. (2004); (49) Mereghetti et al. (2012); (50) Israel et al. (2004b); (51) Guidorzi et al. (2005); (52) Qiu et al. (2005); (53) Rosen et al. (2005); (54) Bloom (2005)

Note. - We do not distinguish between the standard filters listed and any other ones such as $K, K^{\prime}, z^{\prime}, r^{\prime}$, etc. See Table 3 of the online catalog or the original references for further information.

a Counterpart is unconfirmed.

b The originally proposed counterpart has been disputed by Testa et al. (2008).

c Pulsations have been detected in this waveband. 
Table 5

Radio and Mid-Infrared Observations of Magnetars

\begin{tabular}{|c|c|c|c|c|c|c|c|c|c|}
\hline \multirow[b]{2}{*}{ Name } & \multirow[b]{2}{*}{$\begin{array}{l}\text { Detection Frequencies } \\
\qquad(\mathrm{GHz})\end{array}$} & \multicolumn{2}{|c|}{ Radio } & \multirow[b]{2}{*}{$\begin{array}{l}S_{2.0 \mathrm{GHz}} \\
(\mu \mathrm{Jy})\end{array}$} & \multirow[b]{2}{*}{ References } & \multicolumn{4}{|c|}{ Mid-Infrared } \\
\hline & & $\begin{array}{c}\mathrm{DM} \\
\left(\mathrm{cm}^{-3} \mathrm{pc}\right)\end{array}$ & $\begin{array}{l}S_{1.4 \mathrm{GHz}} \\
(\mu \mathrm{Jy})\end{array}$ & & & $\begin{array}{c}F_{4.5 \mu \mathrm{m}} \\
(\mu \mathrm{Jy})\end{array}$ & $\begin{array}{c}F_{8.0 \mu \mathrm{m}} \\
(\mu \mathrm{Jy})\end{array}$ & $\begin{array}{c}F_{24 \mu \mathrm{m}} \\
(\mu \mathrm{Jy})\end{array}$ & References \\
\hline CXOU J010043.1-721134 & 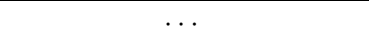 & $\cdots$ & . & $\cdots$ & $\cdots$ & $\ldots$ & $\ldots$ & $\cdots$ & $\ldots$ \\
\hline $4 \mathrm{U} 0142+61$ & 0.11 & 27 & $<46$ & $<4.5$ & $1-3$ & $32.1(2.0)$ & $59.8(8.5)$ & $<38$ & 22,23 \\
\hline SGR $0418+5729$ & $\cdots$ & $\cdots$ & $\cdots$ & $\cdots$ & $\cdots$ & $\ldots$ & $\ldots$ & $\cdots$ & $\cdots$ \\
\hline SGR $0501+4516$ & $\cdots$ & $\cdots$ & $\cdots$ & $<40$ & 4 & $\ldots$ & $\cdots$ & $\ldots$ & $\cdots$ \\
\hline SGR 0526-66 & $\ldots$ & $\ldots$ & $\ldots$ & $\ldots$ & $\cdots$ & $\ldots$ & $\ldots$ & $\ldots$ & $\ldots$ \\
\hline 1E 1048.1-5937 & $\cdots$ & $\cdots$ & $<20$ & $\cdots$ & 5 & $<5.2$ & $<21.8$ & $<39$ & 24,25 \\
\hline 1E $1547.0-5408$ & $1.4-8.6,18.5,43,45$ & $830(50)$ & $<500-4400^{\mathrm{a}}$ & $\cdots$ & 6,7 & $\ldots$ & $\ldots$ & $\ldots$ & $\cdots$ \\
\hline PSR J1622-4950 & $1.4-9.0,17,24$ & $820(30)$ & $<1200-16500^{\mathrm{a}}$ & $\cdots$ & $8-10$ & $\cdots$ & $\cdots$ & $\cdots$ & $\ldots$ \\
\hline SGR $1627-41$ & & & $<80$ & $\cdots$ & 11 & .. & . & $\cdots$ & .. \\
\hline CXOU J164710.2-455216 & $\cdots$ & $\cdots$ & $<40$ & $\cdots$ & 12 & $\ldots$ & $\ldots$ & $\ldots$ & $\ldots$ \\
\hline 1RXS J170849.0-400910 & $\ldots$ & $\ldots$ & $<20$ & $\ldots$ & 5 & $<120$ & $<170$ & $<590$ & 24 \\
\hline CXOU J171405.7-381031 & & $\ldots$ & & ( & & $\cdots$ & $\ldots$ & $\ldots$ & $\cdots$ \\
\hline SGR J1745-2900 & $1.2-8.9,14.6-20,22$ & $1778(3)$ & $\sim 90$ & $\sim 200$ & $13-16$ & $\ldots$ & $\ldots$ & $\ldots$ & $\ldots$ \\
\hline SGR $1806-20$ & & & & $<6.9$ & 2 & $\cdots$ & $\cdots$ & $\cdots$ & $\cdots$ \\
\hline XTE J1810-197 & $0.06,0.35-19,42,88.5,144$ & $178(5)$ & $<150-13600^{\mathrm{a}}$ & $\cdots$ & $17,18,3$ & $<23$ & $<130$ & $<880$ & 24 \\
\hline Swift J1822.3-1606 & & - & & $<50$ & 19 & $\cdots$ & $\ldots$ & $\cdots$ & $\cdots$ \\
\hline SGR 1833-0832 & $\cdots$ & $\cdots$ & $<90$ & $\cdots$ & 20 & $\cdots$ & $\ldots$ & $\ldots$ & $\ldots$ \\
\hline Swift J1834.9-0846 & $\ldots$ & $\ldots$ & $<220$ & $<50$ & 21 & $\ldots$ & $\ldots$ & $\ldots$ & $\ldots$ \\
\hline $1 \mathrm{E} 1841-045$ & $\ldots$ & $\cdots$ & $<20$ & $<10.2$ & 5,2 & $\ldots$ & $\ldots$ & $\ldots$ & $\ldots$ \\
\hline SGR $1900+14$ & $\ldots$ & $\ldots$ & $\cdots$ & $<7.1$ & 2 & $\ldots$ & $\ldots$ & $\ldots$ & $\ldots$ \\
\hline 1E $2259+586$ & $0.06,0.11$ & 79 & $\ldots$ & $<10.8$ & 2,3 & $6.3(1.0)$ & $<20$ & $\ldots$ & 26 \\
\hline SGR $1801-23$ & $\cdots$ & $\cdots$ & $\cdots$ & $\cdots$ & $\cdots$ & $\cdots$ & $\cdots$ & $\cdots$ & $\cdots$ \\
\hline SGR $1808-20$ & $\ldots$ & $\cdots$ & $\cdots$ & $\cdots$ & $\ldots$ & $\cdots$ & $\cdots$ & $\cdots$ & $\ldots$ \\
\hline AX J1818.8-1559 & $\ldots$ & $\ldots$ & $\ldots$ & $\ldots$ & $\ldots$ & $\ldots$ & $\ldots$ & $\ldots$ & $\ldots$ \\
\hline AX $1845.0-0258$ & $\ldots$ & $\ldots$ & $<20$ & $<9.2$ & 5,2 & $\ldots$ & $\ldots$ & $\ldots$ & $\ldots$ \\
\hline SGR $2013+34$ & $\ldots$ & $\ldots$ & & $<9.7$ & 2 & $\ldots$ & $\ldots$ & $\ldots$ & $\ldots$ \\
\hline
\end{tabular}

References. - (1) den Hartog et al. (2007); (2) Lazarus et al. (2012); (3) Malofeev et al. (2012); (4) Hessels et al. (2008); (5) Crawford et al. (2007); (6) Camilo et al. (2007a); (7) Camilo et al. (2008); (8) Levin et al. (2010); (9) Keith et al. (2011); (10) Anderson et al. (2012); (11) Esposito et al. (2009a); (12) Burgay et al. (2006a); (13) Shannon \& Johnston (2013); (14) Eatough et al. (2013); (15) [Spitler et al. (2014); (16) Palaniswamy et al. (2013); (17) [Camilo et al. (2006); (18) Camilo et al. (2007c); (19) Rea et al. (2012b); (20) Esposito et al. (2011); (21) Esposito et al. (2013); (22) Wang \& Kaspi (2008); (23) Wang et al. (2008b); (24) Wang et al. (2007); (25) Wang et al. (2008a); (26) Kaplan et al. (2009a )

a Since these sources are not always visible in radio, the flux densities here range from the lowest reported upper limit for a non-detection to the highest detected value. 
Table 6

Hard X-ray and Gamma-Ray Observations of Magnetars

\begin{tabular}{|c|c|c|c|c|c|c|c|c|c|}
\hline \multirow[b]{3}{*}{ Name } & \multirow[b]{3}{*}{ Telescope $^{\mathrm{b}}$} & \multicolumn{4}{|c|}{ Hard X-ray Spectral Parameters } & \multirow[b]{3}{*}{$E_{\text {cut }}(\mathrm{keV})$} & \multirow[b]{3}{*}{ References } & \multirow{3}{*}{\multicolumn{2}{|c|}{$\begin{array}{l}\text { Gamma Ray } \\
F_{0.1-10 \mathrm{GeV}^{\mathrm{a}}}\end{array}$}} \\
\hline & & \multicolumn{2}{|c|}{ Pulsed Emission } & \multicolumn{2}{|c|}{ Total Emission } & & & & \\
\hline & & $\Gamma^{p}$ & $F_{20-150 \mathrm{keV}}^{p}$ & $\Gamma^{t}$ & $F_{20-150 \mathrm{keV}}^{t}{ }^{\mathrm{C}}$ & & & & \\
\hline CXOU J010043.1-721134 & $\ldots$ & .. & $\ldots$ & $\ldots$ & $\ldots$ & $\ldots$ & $\ldots$ & .. & \\
\hline $4 \mathrm{U} 0142+61$ & $\mathrm{R}, \mathrm{I}$ & $0.40(15)$ & $2.68(1.34)$ & $0.93(6)$ & $9.09(35)$ & $279_{-41}^{+65}$ & 1 & $<0.9$ & \\
\hline & $\mathrm{S}$ & $\ldots$ & $\ldots$ & $0.89_{-0.10}^{+0.11}$ & $\sim 10.3$ & & 2 & & \\
\hline SGR $0418+5729$ & $\ldots$ & $\ldots$ & $\ldots$ & $\ldots .10$ & $\ldots$ & $\ldots$ & $\ldots$ & $<0.4$ & \\
\hline SGR $0501+4516$ & $\mathrm{I}, \mathrm{S}$ & $\ldots$ & $\ldots$ & $0.79_{-0.16}^{+0.20}$ & $<3.5,8.4_{-1.5}^{+2.0}$ & $>100$ & 3 & $<1.9$ & \\
\hline SGR 0526-66 & $\ldots$ & .. & $\ldots$ & $\ldots$ & $\ldots$ & $\ldots$ & $\ldots$ & $\ldots$ & \\
\hline $1 \mathrm{E} 1048.1-5937^{\mathrm{d}}$ & $\cdots$ & $\cdots$ & $\ldots$ & $\cdots$ & $\ldots$ & $\ldots$ & $\ldots$ & $<5.3$ & \\
\hline 1E $1547.0-5408$ & $\mathrm{R}, \mathrm{I}$ & $-\left(0.37_{-0.20}^{+0.28}-1.55_{-0.26}^{+0.42}\right)$ & $4.1(9)-7.5_{-1.0}^{+0.9}$ & $0.87(7)-1.45(4)$ & $<1.5,8.0(2.2)-25.2(3.7)$ & & 5 & $<10.0$ & \\
\hline & $\mathrm{S}$ & $\ldots$ & $\ldots$ & $1.54_{-0.05}^{+0.06}$ & $17.4_{-1.8}^{+1.4}$ & $>200$ & 6 & & \\
\hline PSR J1622-4950 & $\ldots$ & .. & $\ldots$ & $\ldots$ & $\ldots$ & $\ldots$ & $\ldots$ & . & \\
\hline SGR $1627-41$ & $\cdots$ & $\cdots$ & $\ldots$ & $\ldots$ & $\ldots$ & $\ldots$ & $\ldots$ & $<20.0$ & \\
\hline CXOU J164710.2-455216 & $\ldots$ & $\ldots$ & $\ldots$ & $\ldots$ & $\ldots$ & $\ldots$ & $\ldots$ & $<10.0$ & \\
\hline 1RXS J170849.0-400910 & $\mathrm{R}, \mathrm{I}$ & $0.86(16)$ & $2.60(35)$ & $1.13(6), 1.46(21)$ & $5.2(1.0), 6.61(23)$ & $>300$ & 7,8 & $<10.0$ & \\
\hline CXOU J171405.7-381031 & $\cdots$ & $\cdots$ & .. & & & $\ldots$ & $\cdots$ & $\cdots$ & \\
\hline SGR J1745-2900 & $\mathrm{N}$ & .. & $\ldots$ & $1.47_{-0.37}^{+0.46}$ & $0.67_{-0.27}^{+0.20}$ & $>50$ & 9 & $\ldots$ & \\
\hline SGR $1806-20$ & I & $\cdots$ & $\cdots$ & $1.5(3), 1.9(2)$ & $6.0(9), 11(2)$ & $>160$ & 10,11 & $<0.6$ & $\bigcirc$ \\
\hline & $\mathrm{S}$ & $\cdots$ & $\cdots$ & $1.2(1)-1.7(1)$ & $\sim 3.8-9.9$ & & 12 & & \\
\hline XTE J1810-197 & $\ldots$ & $\ldots$ & $\ldots$ & $\ldots$ & $\ldots$ & $\ldots$ & $\ldots$ & $<5.0$ & c \\
\hline Swift J1822.3-1606 & $\cdots$ & $\cdots$ & $\cdots$ & $\cdots$ & $\cdots$ & $\cdots$ & $\cdots$ & $\ldots$ & In \\
\hline SGR $1833-0832$ & $\ldots$ & $\ldots$ & $\ldots$ & $\ldots$ & $\ldots$ & $\ldots$ & $\ldots$ & $\ldots$ & 䜤 \\
\hline Swift J1834.9-0846 & $\ldots$ & $\ldots$ & $\ldots$ & $\ldots$ & $\cdots$ & $\cdots$ & $\cdots$ & $\cdots$ & $\infty$ \\
\hline $1 \mathrm{E} 1841-045$ & I & $0.72(15)$ & $\sim 4.0$ & $1.32(11)$ & $\sim 6.9$ & $>140$ & 13 & $<3.0$ & 4 \\
\hline & $\mathrm{S}$ & $1.35_{-0.30}^{+0.30}$ & $\sim 2.7$ & $1.62_{-0.21}^{+0.21}$ & $\sim 4.6$ & & 14 & & $\Sigma$ \\
\hline & $\mathrm{N}$ & $0.99(36)$ & $\sim 3.0$ & $1.33(3)$ & $\sim 8.0$ & & 15 & & \\
\hline SGR $1900+14$ & I & $\ldots$ & $\ldots$ & $3.1(5)$ & $1.6(4)$ & $>100$ & 16 & $<0.4$ & Z \\
\hline & $\mathrm{S}$ & $\ldots$ & $\ldots$ & $1.2(5)-1.4(3)$ & $\sim 1.4-3.2$ & & 12 & & \\
\hline 1E $2259+586$ & $\mathrm{R}, \mathrm{S}$ & $-1.02(24)$ & $\sim 5.9^{\mathrm{e}}$ & ... & $<2.0$ & $\ldots$ & 13,12 & $<1.7$ & \\
\hline SGR $1801-23$ & & & & & & & & & \\
\hline SGR $1808-20$ & $\ldots$ & $\ldots$ & $\ldots$ & $\ldots$ & $\ldots$ & $\ldots$ & $\ldots$ & $\ldots$ & \\
\hline AX J1818.8-1559 & $\ldots$ & $\cdots$ & $\ldots$ & $\ldots$ & $\ldots$ & $\ldots$ & $\ldots$ & $\cdots$ & \\
\hline AX $1845.0-0258$ & $\ldots$ & $\ldots$ & $\ldots$ & $\ldots$ & $\ldots$ & $\ldots$ & $\ldots$ & $\ldots$ & \\
\hline SGR $2013+34$ & $\ldots$ & $\ldots$ & $\ldots$ & $\ldots$ & $\ldots$ & $\ldots$ & $\ldots$ & $\ldots$ & \\
\hline
\end{tabular}

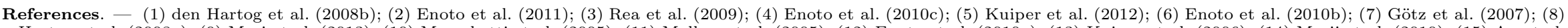

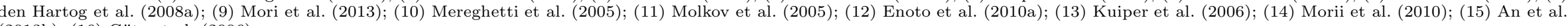
(2013b); (16) Götz et al. (2006)

Note. - Values in italics were measured when the source was in outburst.

a Gamma ray flux upper limits are taken from Abdo et al. (2010).

b R: RXTE, I: Integral, S: Suzaku, N: NuSTAR.

${ }^{c}$ Hard X-ray and gamma ray fluxes are in units of $10^{-11} \mathrm{erg} \mathrm{s}^{-1} \mathrm{~cm}^{-2}$

d 1E 1048.1-5937 was detected in hard X-rays with INTEGRAL by Leyder et al. (2008), but no spectral information was given.

e Pulsed emission from 1E 2259+586 was only observed by RXTE up to $\sim 25 \mathrm{keV}$, so the extrapolated $20-150 \mathrm{keV}$ pulsed flux should not be considered reliable. 
Table 7

Magnetar Associations and Distances

\begin{tabular}{|c|c|c|c|c|c|c|c|c|}
\hline Name & Proposed Associations & $\begin{array}{l}\text { SNR Age } \\
\text { (kyr) }\end{array}$ & References & $\begin{array}{l}\text { Distance } \\
(\mathrm{kpc})\end{array}$ & Measured To & Reference & $\begin{array}{c}z \\
(\mathrm{pc})\end{array}$ & $L_{\mathrm{X}}^{\mathrm{a}}$ \\
\hline CXOU J010043.1-721134 & $\mathrm{SMC}$ & $\ldots$ & 1 & $62.4(1.6)$ & SMC & 28 & $\ldots$ & 65 \\
\hline $4 \mathrm{U} 0142+61$ & $\ldots$ & $\ldots$ & $\ldots$ & $3.6(4)$ & $0142+61$ & 29 & $-27(3)$ & 105 \\
\hline SGR $0418+5729$ & $\ldots$ & $\ldots$ & $\ldots$ & $\sim 2$ & Perseus Arm & 30 & $\sim 180$ & 0.00096 \\
\hline SGR $0501+4516$ & SNR HB $9^{\mathrm{b}}$ & $4-7$ & 2,3 & $\sim 2$ & Perseus Arm & 31 & $\sim 68$ & 0.40 \\
\hline SGR 0526-66 & LMC, SNR N49b, SL 463 & $\sim 4.8$ & $4-6$ & $53.6(1.2)$ & LMC & 32 & $\ldots$ & 189 \\
\hline 1E $1048.1-5937$ & GSH $288.3-0.5-28^{\mathrm{b}}$ & $\ldots$ & 7 & $9.0(1.7)$ & $1048.1-5937$ & 29 & $-82(15)$ & 49 \\
\hline 1E $1547.0-5408$ & SNR G327.24-0.13 & $\ldots$ & 8 & $4.5(5)$ & $1547.0-5408$ & 33 & $-10.3(1.1)$ & 1.3 \\
\hline PSR J1622-4950 & SNR G333.9+0.0 & $<6$ & 9 & $\sim 9$ & J1622-4950 & 34 & $\sim-16$ & 0.44 \\
\hline SGR $1627-41$ & CTB 33, MC -71, SNR G337.0-0.1 & $\ldots$ & 10,11 & $11.0(3)$ & G337.0-0.1 & 11 & $-21.4(6)$ & 3.6 \\
\hline CXOU J164710.2-455216 & Westerlund 1 & $\ldots$ & 12 & $3.9(7)$ & Westerlund 1 & 35 & $-29(5)$ & 0.45 \\
\hline 1RXS J170849.0-400910 & & $\ldots$ & $\ldots$ & $3.8(5)$ & J170849.0-400910 & 29 & $2.4(3)$ & 42 \\
\hline CXOU J171405.7-381031 & SNR CTB 37B & $0.65_{-0.30}^{+2.50}$ & 13,14 & $\sim 13.2$ & CTB 37B & 36 & $\sim 86$ & 56 \\
\hline SGR J1745-2900 & Galactic Center & $\cdots$ & 15 & $\sim 8.5$ & Galactic Center & 37 & $\sim-7.0$ & $<0.11$ \\
\hline SGR $1806-20$ & W31, MC 13A, Star cluster & $\cdots$ & 16,17 & $8.7_{-1.5}^{+1.8}$ & Star cluster & 38 & $-36.7_{-7.6}^{+6.3}$ & 163 \\
\hline XTE J1810-197 & $\cdots$ & $\cdots$ & $\ldots$ & $3.5_{-0.4}^{+0.5}$ & J1810-197 & 39 & $-9.7_{-1.4}^{+1.1}$ & 0.043 \\
\hline Swift J1822.3-1606 & M17 & $\ldots$ & 18 & $\begin{array}{l}-0.4 \\
1.6(3)\end{array}$ & M17 & 18 & $-28.5(5.3)$ & $<0.0077$ \\
\hline SGR $1833-0832$ & & & & & & & $\sim 3.6$ & $<2.4$ \\
\hline Swift J1834.9-0846 & SNR W41 & $\sim 100$ & 19,20 & $4.2(3)$ & W41 & 40 & $-25(2)$ & $<0.0084$ \\
\hline 1E $1841-045$ & SNR Kes 73 & $0.5-1$ & 21,22 & $8.5_{-1.0}^{+1.3}$ & Kes 73 & 22 & $-0.97_{-0.15}^{+0.11}$ & 184 \\
\hline SGR $1900+14$ & Star cluster & & 23 & $12.5(1.7)$ & Star cluster & 41 & $167(23)$ & 90 \\
\hline $1 \mathrm{E} 2259+586$ & SNR CTB 109 & $14(2)$ & 24,25 & $3.2(2)$ & CTB 109 & 42 & $-55.6(3.5)$ & 17 \\
\hline SGR 1801-23 & 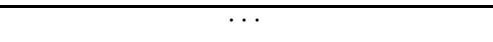 & $\cdots$ & $\cdots$ & $\cdots$ & $\cdots$ & $\cdots$ & $\sim 12$ & \\
\hline SGR $1808-20$ & $\ldots$ & $\cdots$ & $\ldots$ & $\ldots$ & $\ldots$ & $\cdots$ & $\sim-45$ & \\
\hline AX J1818.8-1559 & $\ldots$ & $\ldots$ & $\ldots$ & $\ldots$ & $\ldots$ & $\cdots$ & $\sim-44$ & 20 \\
\hline AX $1845.0-0258$ & SNR G29.6+0.1 & $<8$ & 26 & $\sim 8.5$ & Scutum Arm & 43 & $\sim 16$ & 2.9 \\
\hline SGR $2013+34$ & W58 & $\cdots$ & 27 & $\sim 8.8$ & W58 & 27 & $\sim-16$ & \\
\hline
\end{tabular}

References. - (1) Lamb et al. (2002); (2) Gaensler \& Chatteriee (2008); (3) Leahy \& Tian (2007); (4) Cline et al. (1982); (5) Klose et al. (2004); (6) Park et al. (2012); (7) Gaensler et al. (2005); (8) Gelfand \& Gaensler (2007); (9) Anderson et al. (2012); (10) Woods et al. (1999); (11) Corbel et al. (1999); (12) Muno et al. (2006); (13) (20) Fuchs et al. (1999); (17) Corbel \& Eikenberry (2004); (18) Scholz et al. (2012); (19) Tian et al. (2007); (20) Kargaltsev et al. 2012); (21) Vasisht \& Gotthelf (1997); (22) Tian \& Leahy (2008); (23) Vrba et al. (2000); (24) Fahlman \& Gregory (1981); (25) a

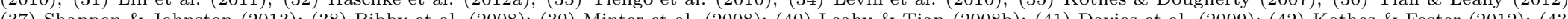
(37) Shannon \& Johnston (2013); (38) Bibbv et al. (2008); (39) Minter et al. (2008); (40) Leahy \& Tian (2008b); (41) Davies et al. (2009); (42) Kothes \& Foster (2012); (43)

$2-10 \mathrm{keV}$ X-ray luminosity in units of $10^{33} \mathrm{erg} \mathrm{s}^{-1}$. No uncertainties have been included.

b The proposed association with this source has been disputed 
Table 8

Magnetar Names

\begin{tabular}{|c|c|c|c|}
\hline Current Name & Alternate Current Name & MG Name & ATNF (PSR) Name \\
\hline CXOU J010043.1-721134 & $\ldots$ & MG J0100-7211 & PSR J0100-7211 \\
\hline $4 \mathrm{U} 0142+61$ & $\ldots$ & MG J0146+6145 & PSR J0146+6145 \\
\hline SGR $0418+5729$ & $\ldots$ & MG J0418+5732 & PSR J0418+5732 \\
\hline SGR $0501+4516$ & $\cdots$ & MG J0501+4516 & PSR J0501+4516 \\
\hline SGR 0526-66 & $\ldots$ & MG J0526-6604 & PSR J0525-6607 \\
\hline 1E $1048.1-5937$ & $\ldots$ & MG J1050-5953 & PSR J1048-5937 \\
\hline $1 \mathrm{E} 1547.0-5408$ & SGR J1550-5418 & MG J1550-5418 & PSR J1550-5418 \\
\hline PSR J1622-4950 & $\ldots$ & MG J1622-4950 & PSR J1622-4950 \\
\hline SGR $1627-41$ & $\ldots$ & MG J1635-4735 & PSR J1635-4735 \\
\hline CXOU J164710.2-455216 & $\cdots$ & MG J1647-4552 & PSR J1647-4552 \\
\hline 1RXS J170849.0-400910 & $\ldots$ & MG J1708-4008 & PSR J1708-4009 \\
\hline CXOU J171405.7-381031 & $\cdots$ & MG J1714-3810 & PSR J1714-3810 \\
\hline SGR J1745-2900 & SGR J1745-29 & MG J1745-2900 & PSR J1745-2900 \\
\hline SGR $1806-20$ & $\ldots$ & MG J1808-2024 & PSR J1808-2024 \\
\hline XTE J1810-197 & $\ldots$ & MG J1809-1943 & PSR J1809-1943 \\
\hline Swift J1822.3-1606 & $\ldots$ & MG J1822-1604 & PSR J1822-1606 \\
\hline SGR 1833-0832 & $\ldots$ & MG J1833-0831 & PSR J1833-0831 \\
\hline Swift J1834.9-0846 & $\ldots$ & MG J1834-0845 & PSR J1834-0845 \\
\hline 1E $1841-045$ & $\ldots$ & MG J1841-0456 & PSR J1841-0456 \\
\hline SGR $1900+14$ & $\ldots$ & MG J1907+0919 & PSR J1907+0919 \\
\hline $1 \mathrm{E} 2259+586$ & $\ldots$ & MG J2301+5852 & PSR J2301+5852 \\
\hline SGR 1801-23 & $\cdots$ & $\cdots$ & $\cdots$ \\
\hline SGR $1808-20$ & & $\ldots$ & $\ldots$ \\
\hline AX J1818.8-1559 & GRB 071017 & $\ldots$ & $\ldots$ \\
\hline AX $1845.0-0258$ & & $\ldots$ & PSR J1845-0256 \\
\hline SGR $2013+34$ & GRB 050925 & $\cdots$ & ... \\
\hline
\end{tabular}

